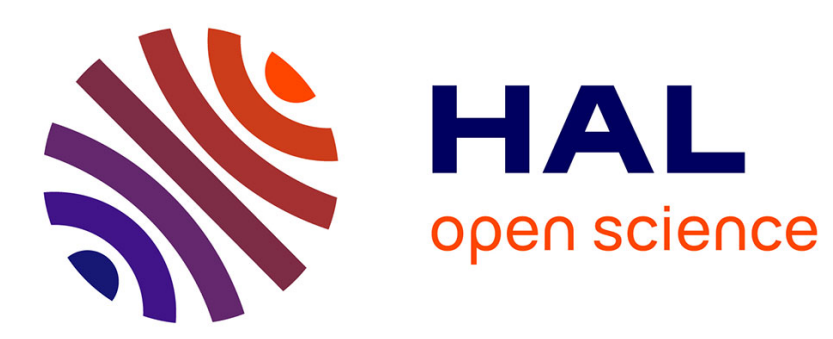

\title{
A fractional step method to compute a class of compressible gas-liquid flows
}

Jean-Marc Hérard, Olivier Hurisse

\section{To cite this version:}

Jean-Marc Hérard, Olivier Hurisse. A fractional step method to compute a class of compressible gas-liquid flows. Computers and Fluids, 2012, 55, pp.57-69. 10.1016/j.compfluid.2011.11.001 . hal01265315

\section{HAL Id: hal-01265315 \\ https://hal.science/hal-01265315}

Submitted on 1 Feb 2016

HAL is a multi-disciplinary open access archive for the deposit and dissemination of scientific research documents, whether they are published or not. The documents may come from teaching and research institutions in France or abroad, or from public or private research centers.
L'archive ouverte pluridisciplinaire HAL, est destinée au dépôt et à la diffusion de documents scientifiques de niveau recherche, publiés ou non, émanant des établissements d'enseignement et de recherche français ou étrangers, des laboratoires publics ou privés. 


\title{
A fractional step method to compute a class of compressible gas-liquid flows
}

\author{
Jean-Marc Hérard* Olivier Hurisse ${ }^{\dagger}$ \\ $E D F, R \& D \quad E D F, R E D$
}

\begin{abstract}
We present in this paper some algorithms dedicated to the computation of numerical approximations of a class of two-fluid two-phase flow models. Governing equations for the statistical void fraction, partial mass, momentum, energy are presented first, and meaningful closure laws are given. Then we may give the main properties of the class of two-fluid models. The whole algorithm that relies on the fractional step method and complies with the entropy inequality is presented afterwards. Emphasis is given on the computation of pressure-velocity-temperature relaxation source terms. Conditions pertaining to the existence and uniqueness of discrete solutions of the relaxation step are given. While focusing on some one-dimensional test cases, the true rates of convergence may be obtained within the evolution step and the relaxation step. Eventually, some twodimensional numerical simulations of a heated wall are shown and are briefly discussed. Some advantages and weaknesses of algorithms are also discussed.
\end{abstract}

Keywords:

Two-phase flows / Finite Volume schemes / Two-fluid model / Hyperbolic systems / Closure laws / Entropy inequality / Relaxation effects.

\section{Introduction}

Two distinct types of models are used in order to compute liquid-gas or watervapour two-phase flows in industrial codes: the homogeneous approach and the two-fluid approach. Within the framework of nuclear safety codes[16] , the homogeneous approach is generally adopted for codes dedicated to components such as reactor cores and steam generators (THYC, FLICA and GENEPI codes in France), whereas the two-fluid approach is prefered in system codes

\footnotetext{
${ }^{*}$ Corresponding author. EDF, R\&D, Fluid Dynamics, Power Generation and Environment, 6 quai Watier, 78400, Chatou, France. Tel: (33)-1-30 8770 37. Email: jeanmarc.herard@edf.fr

${ }^{\dagger}$ EDF, R\&D, Fluid Dynamics, Power Generation and Environment, 6 quai Watier, 78400, Chatou, France.
} 
(CATHARE or RELAP codes for instance) and also in 3D commercial codes (CFX, Star-CD, Fluent) and inhouse codes (NEPTUNE-CFD). The two-fluid approach is assumed to be more general, and it is also expected to predict more accurately flows for which the phasic desequilibrium plays a crucial role (see $[9,25,26])$.

Now, two distinct two-fluid approaches may be considered. The first one, which is the most standard one, relies on an instantaneous pressure equilibrium between both phases. The second one no longer assumes this hypothesis, which means that the seven unknowns corresponding to the statistical fraction of liquid, the two mean velocities, the two mean temperatures and the two mean pressures are evaluated by searching approximations of solutions of a coupled set of seven partial differential equations (PDE). These equations correspond to the mass balance, the momentum balance and the energy balance within each phase, and is supplemented by the governing equation for the statistical liquid fraction.

This paper is devoted to the simulation of water-gas flow models belonging to the second class. Though this approach was introduced approximately thirty years ago (see [33]), few workers have been investigating this class until the late 90's. Within the framework of gas-particle flows, and more precisely when studying Deflagration to Detonation Transition, these models gained a considerable interest within a small community. Among other studies, one should at least point out the contributions $[4,28,5,27,32]$ which are concerned with the modeling aspects. Fewer papers tackle the problem of water-gas or water-vapour flows, among which we must quote [33, 34] and more recently the article [12] , that examines a medium of small oscillating bubbles in a liquid medium, and also provides a general formalism in order to derive meaningful governing equations. A classification of closure laws related to the interface pressure $P_{I}$ and the interface velocity $V_{I}$ was proposed in $[8,11]$, which provides a general framework relying on two main ingredients:

- (H1) the interface velocity $V_{I}$, which governs the evolution of the statistical void fraction, should be such that the field associated with the eigenvalue $\lambda=V_{I}$ were linearly degenerate;

- (H2) a physically relevant entropy inequality should control smooth solutions.

Based on these two keystones, it has been shown in $[8,11]$ that one may retrieve the well-known Baer-Nunziato model, that corresponds to the particular choice $P_{I}=P_{l}, V_{I}=U_{g}$, among other possibilities (where $P_{l}$ and $U_{g}$ respectively refer to the liquid pressure and the gas velocity). We emphasize that this approach was recently extended to the framework of two-phase flow in porous media $[19,13]$. The same procedure provides some way to tackle the modeling of three-phase flows[18] . It also gives a relevant approach for the modeling of dense granular flows[10]. These extensions confirm the relevance of the whole 
modeling approach. However, one must be aware that at least two difficulties are hidden in these sets of PDE.

First, the convective part of the system of PDE is hyperbolic with no constraining condition on the physical states, but it contains two (or three, depending on the closure law for $V_{I}$ ) linearly degenerate fields. A straightforward consequence is that the asymptotic rate of convergence of so-called first-order (respectively second-order) Riemann solvers is $1 / 2$ (respectively $2 / 3$ ). This has recently motivated great efforts in order to build accurate enough Riemann solvers for the Baer-Nunziato system (see at least $[1,2,7,31,35,36]$ ). Second, the system contains stiff source terms which are linked with the pressurevelocity-temperature relaxation effects. These require specific algorithms, and the main part of the present paper is actually dedicated to this work. This is basically motivated by the fact that few available articles discuss these tricky problems. In the sequel, the two-fluid two-pressure model accounts for velocity, pressure and temperature relaxation, each one being associated with a non-zero time scale. Actually, most of the papers in the literature focus on some specific situations. Dealing with the velocity relaxation effects is not really challenging, and a lot of schemes have been proposed in order to cope with it. On the contrary, there are few articles dealing with the numerical treatment of the pressure relaxation. In the case of instantaneous relaxation (i.e. with "zero time scales") one can refer to [34] (which deals with instantaneous velocity and pressure relaxation) or [21] (which only deals with instantaneous pressurerelaxation) among others. For non-zero pressure relaxation time scales, a scheme has been proposed in [11], with an important drawback : the total energy of the mixture is not conserved by the scheme. Moreover, most of the references in the literature are based on models where the temperature relaxation source terms are neglected, and consequently there are no numerical schemes accounting for them.

Hence the paper is organized as follows. We first recall the set of PDEs that governs the two-fluid model, and recall its main properties. Next we present approximate Riemann solvers and algorithms used to compute approximations of solutions of the coupled ODEs arising when taking relaxation effects into account. The most difficult task dwells in the building of suitable algorithms in the pressure relaxation step. Of course this difficulty vanishes when the pressure relaxation time scale is set to zero, but in that case convergence difficulties -with respect to the mesh size- may be expected (see [21, 17, 10]) when focusing on unsteady computations. The first section of numerical results focuses on the practical estimation of the rate of convergence when looking for approximate solutions of the convective subset. The second section gives emphasis on the approximation of velocity-pressure-temperature relaxation effects. The last section is devoted to the two-dimensional simulation of the flow close to a heated wall. 


\section{Governing equations and main properties of the two-fluid model}

\subsection{The two-fluid model}

Throughout the paper, indexes $l, g$ refer to the liquid and gas phases; the statistical void fractions of gas and liquid are noted classically $\alpha_{g}$ and $\alpha_{l}$, which should agree with:

$$
\alpha_{l}+\alpha_{g}=1
$$

The mean pressures, mean velocities and mean densities of the two phases are denoted $P_{\phi}, U_{\phi}$ and $\rho_{\phi}$ respectively, for $\phi=l, g$. The total energy within each phase is:

$$
E_{\phi}=\rho_{\phi} e_{\phi}\left(P_{\phi}, \rho_{\phi}\right)+\rho_{\phi} \frac{U_{\phi}^{2}}{2}, \quad \phi=g, l
$$

Internal energy functions $e_{\phi}$ are provided by users.

The so-called conservative variable $W$ will be defined as:

$$
W=\left(\alpha_{l}, \alpha_{l} \rho_{l}, \alpha_{l} \rho_{l} U_{l}, \alpha_{l} E_{l}, \alpha_{g} \rho_{g}, \alpha_{g} \rho_{g} U_{g}, \alpha_{g} E_{g}\right)
$$

Moreover, $P_{I}(W)$ and $V_{I}(W)$ respectively denote in this paper the interfacial pressure and velocity, and will precised afterwards. These interface terms $V_{I}$ and $P_{I}$ will be such that:

- jump conditions are well defined within each isolated field;

- a physically relevant entropy inequality holds for smooth solutions of (2).

Given these notations, the governing set of equations for first-order moments may be written as follows in a multi-dimensional framework:

$$
\left\{\begin{array}{l}
\partial_{t}\left(\alpha_{l}\right)+V_{I} \nabla \alpha_{l}=S_{1, l} \\
\partial_{t}\left(\alpha_{l} \rho_{l}\right)+\nabla \cdot\left(\alpha_{l} \rho_{l} U_{l}\right)=S_{2, l} \\
\partial_{t}\left(\alpha_{l} \rho_{l} U_{l}\right)+\nabla \cdot\left(\alpha_{l} \rho_{l} U_{l} \otimes U_{l}+\alpha_{l} P_{l} \mathcal{I}\right)-P_{I} \nabla\left(\alpha_{l}\right)=S_{3, l} \\
\partial_{t}\left(\alpha_{l} E_{l}\right)+\nabla \cdot\left(\alpha_{l} U_{l}\left(E_{l}+P_{l}\right)\right)+P_{I} \partial_{t}\left(\alpha_{l}\right)=S_{4, l} \\
\partial_{t}\left(\alpha_{g} \rho_{g}\right)+\nabla \cdot\left(\alpha_{g} \rho_{g} U_{g}\right)=-S_{2, l} \\
\partial_{t}\left(\alpha_{g} \rho_{g} U_{g}\right)+\nabla \cdot\left(\alpha_{g} \rho_{g} U_{g} \otimes U_{g}+\alpha_{g} P_{g} \mathcal{I}\right)-P_{I} \nabla\left(\alpha_{g}\right)=-S_{3, l} \\
\partial_{t}\left(\alpha_{g} E_{g}\right)+\nabla \cdot\left(\alpha_{g} U_{g}\left(E_{g}+P_{g}\right)\right)+P_{I} \partial_{t}\left(\alpha_{g}\right)=-S_{4, l}
\end{array}\right.
$$

where right-hand side terms $S_{k, l}(W)$ represent the source terms (for $k=2,3,4$ ), which enable to account for mass transfer, momentum and energy transfer through the interface between the two phases. The term $S_{1, l}$ will also be introduced later on. External sources might be included but are not considered herein. A derivation of the first governing equation of the statistical liquid fraction $\alpha_{l}$ can be found in[20]. Standard viscous contributions may be included, which of course comply with the entropy inequality that will be detailed in the 
next subsection. Relevant boundary conditions and initial conditions should also be prescribed.

From now on, partial masses will be noted $m_{\phi}=\alpha_{\phi} \rho_{\phi}$, and we introduce specific entropies $s_{\phi}$ in agreement with the constraint:

$$
\left(c_{\phi}\right)^{2} \partial_{P_{\phi}}\left(s_{\phi}\right)+\partial_{\rho_{\phi}}\left(s_{\phi}\right)=0
$$

and temperatures $T_{\phi}$ as follows:

$$
1 / T_{\phi}=\partial_{P_{\phi}}\left(s_{\phi}\right) / \partial_{P_{\phi}}\left(e_{\phi}\right)
$$

\subsection{Closure laws for interfacial transfer terms}

The interfacial transfer contributions have been studied in $[4,5,17,10,28,29]$, and a summary can be found in appendix A. These terms are thus defined as:

$$
\begin{aligned}
& S_{1, l}=\left(\tau_{2}\right)^{-1} \frac{\alpha_{l} \alpha_{g}}{\left|P_{l}\right|+\left|P_{g}\right|}\left(P_{l}-P_{g}\right) \\
& S_{2, l} \stackrel{\text { def }}{=} \Gamma=\left(\tau_{1}\right)^{-1} \frac{1}{T_{g}^{-1}\left|g_{g}\right|+T_{l}^{-1}\left|g_{l}\right|} \frac{m_{l} m_{g}}{m_{l}+m_{g}}\left(T_{g}^{-1} g_{g}-T_{l}^{-1} g_{l}\right) \\
& S_{3, l}=\mathcal{D}+\left(U_{l}+U_{g}\right) \Gamma / 2 \\
& S_{4, l}=\left(\tau_{4}\right)^{-1} \frac{m_{l} C_{V, l} m_{g} C_{V, g}}{m_{l} C_{V, l}+m_{g} C_{V, g}}\left(T_{g}-T_{l}\right)+\left(U_{l}+U_{g}\right) \mathcal{D} / 2+\left(U_{l} U_{g}\right) \Gamma / 2
\end{aligned}
$$

where free enthalpies $g_{\phi}$ are defined as:

$$
g_{\phi}=\left(e_{\phi}+\frac{P_{\phi}}{\rho_{\phi}}\right)-T_{\phi} s_{\phi}
$$

The drag term $\mathcal{D}$ is modeled according to:

$$
\mathcal{D}=\left(\tau_{3}\right)^{-1} \frac{m_{l} m_{g}}{m_{l}+m_{g}}\left(U_{g}-U_{l}\right)
$$

These closure laws involve -positive- time scales which are noted $\tau_{k}$ (for $k=$ $1, . ., 4)$. We recall that:

$$
C_{V, \phi}=\partial_{T_{\phi}}\left(e_{\phi}\right)_{\mid \rho_{\phi}}, \text { for } \phi=l, g .
$$

Since we focus in this paper on gas-liquid flows, we will no longer consider mass transfer contributions $S_{2, l}$ in the sequel.

\subsection{Closure laws for $\left(P_{I}, V_{I}\right)$}

Actually, a keystone in the approach is a relevant definition of the couple $\left(P_{I}, V_{I}\right)$. We recall that the enforcement of a relevant entropy inequality $(\mathbf{H 2})$ has a straightforward consequence, which is that $P_{I}$ may be written in terms of $V_{I}$ and $W$ in a unique way (see equations $(27),(28),(29)$ in Appendix A). The same holds when tackling three-phase flows, as emphasized in [18]. Now, the second requirement $(\mathbf{H 1})$ implies that the field associated with $\lambda=V_{I}$ should be linearly degenerate. As shown in $[8,11]$, few expressions guarantee this behaviour. Among these, one must point out the following two: 
- The mixture velocity closure, that is: $V_{I}=U_{m} \stackrel{\text { def }}{=}\left(m_{l} U_{l}+m_{g} U_{g}\right) /\left(m_{l}+\right.$ $\left.m_{g}\right)$, and its corresponding value $P_{I}=\mu P_{l}+(1-\mu) P_{g}$, setting $\mu=$ $\frac{1}{1+m_{l} T_{l} / m_{g} T_{g}}$. Thus it corresponds in the asymptotic regime $T_{l}=T_{g}$ to $\left(m_{g} P_{l}+m_{l} P_{g}\right) /\left(m_{g}+m_{l}\right)$, which means that the interface pressure is mainly driven by the pressure of the vanishing phase $P_{g}$ when $m_{g}$ tends to 0 ;

- The so-called Baer-Nunziato closure, which corresponds to the choice $V_{I}=$ $U_{g}$, and consequently $P_{I}=P_{l}$, owing to closure laws (27),(28),(29); this model is well suited for two-phase flows where the gas phase is dilute $\left(\alpha_{g}<<1\right)$.

Actually, an extended framework including the latter two formulations may be exhibited (see [20]), but it is far beyond the scope of the present work.

From now on, we will restrict our attention to the latter couple $\left(P_{I}, V_{I}\right)=$ $\left(P_{l}, U_{g}\right)$.

\subsection{Main properties of the two-fluid model}

We may now recall in brief the main properties of system (2) using the previous closure laws. We emphasize that these are valid the for the above defined closure laws. The reader is refered to the references $[8,11]$ (see also $[18,19])$ that contain all proofs, comments and details. We recall first that the system (2) is invariant under frame rotation, and we provide three important properties below.

- Property 1: (Hyperbolicity and structure of waves)

In a one-dimensional framework, the set of equations (2) is hyperbolic; it admits seven real eigenvalues:

$\lambda_{1,2}=U_{g}, \quad \lambda_{3}=U_{g}-c_{g}, \quad \lambda_{4}=U_{g}+c_{g}, \quad \lambda_{5}=U_{l}, \quad \lambda_{6}=U_{l}-c_{l}, \quad \lambda_{7}=U_{l}+c_{l}$

and associated righteigenvectors span the whole space $\mathcal{R}^{7}$, unless $\mid U_{l}-$ $U_{g} \mid / c_{l}=1$. Fields associated with eigenvalues $\lambda_{1,2,5}$ are linearly degenerate. Other fields are genuinely non linear.

- Property 2: (Jump conditions in a one-dimensional framework)

Within each isolated field associated with $\lambda_{k}$, unique jump conditions hold. Apart from the field associated with the eigenvalue $\lambda=U_{g}, \alpha_{l}$ is uniform and thus these jump conditions correspond to single phase jump relations, that is:

$$
\begin{aligned}
& -\sigma\left[\rho_{\phi}\right]_{L}^{R}+\left[\rho_{\phi} U_{\phi}\right]_{L}^{R}=0 ; \\
& -\sigma\left[\rho_{\phi} U_{\phi}\right]_{L}^{R}+\left[\rho_{\phi} U_{\phi}^{2}+P_{\phi}\right]_{L}^{R}=0 ; \\
& -\sigma\left[E_{\phi}\right]_{L}^{R}+\left[U_{\phi}\left(E_{\phi}+P_{\phi}\right)\right]_{L}^{R}=0,
\end{aligned}
$$

where $\sigma$ denotes the speed of the shock wave, and $L, R$ subscripts refer to the left-right states on each side of this travelling shock wave. 
- Property 3: (Entropy inequality)

Define the entropy $\eta(W)=m_{l} s_{l}+m_{g} s_{g}$ and the entropy flux $f_{\eta}(W)=$ $m_{l} s_{l} U_{l}+m_{g} s_{g} U_{g}$; then smooth solutions $W$ of (2) are such that:

$$
0 \leq \partial_{t}(\eta(W))+\nabla \cdot\left(f_{\eta}(W)\right) .
$$

Obviously, the LD structure of the 1,2-field is crucial in order to obtain unique jump conditions. Otherwise the computation of shock solutions would be meaningless, since multiple shock solutions may be obtained using various -stable- solvers (see for examples [17]) . This point is not clearly adressed in the literature, though of major importance. The entropy inequality enables to select admissible shock waves, and may also be used to build schemes for the computation of convective effects. Obviously, an alternative formulation of jump conditions in genuinely non linear fields that is probably more convenient may be:

$$
\begin{aligned}
& \sigma=\left[\rho_{\phi} U_{\phi}\right]_{L}^{R} /\left[\rho_{\phi}\right]_{L}^{R} ; \\
& \left(\rho_{\phi}\right)_{L}\left(\rho_{\phi}\right)_{R}\left(\left[U_{\phi}\right]_{L}^{R}\right)^{2}=\left[\rho_{\phi}\right]_{L}^{R}\left[P_{\phi}\right]_{L}^{R} ; \\
& \left.2\left(\left[e_{\phi}\right]\right)_{L}^{R}+\left(P_{\phi}\right)_{L}+\left(P_{\phi}\right)_{R}\right)\left[\left(\rho_{\phi}\right)^{-1}\right]_{L}^{R}=0,
\end{aligned}
$$

We now provide the whole algorithm that is used in order to obtain approximate solutions of system (2).

\section{Numerical algorithm}

\subsection{Fractional step method}

We use a fractional step method that complies with the entropy inequality (5);

a first "evolution" step accounts for all convective effects, while the second "relaxation" step takes source terms into account.

- Evolution step

This step computes approximate solutions of the hyperbolic homogeneous system:

$$
\left\{\begin{array}{l}
\partial_{t}\left(\alpha_{l}\right)+V_{I} \nabla \alpha_{l}=0 \\
\partial_{t}\left(\alpha_{l} \rho_{l}\right)+\nabla \cdot\left(\alpha_{l} \rho_{l} U_{l}\right)=0 \\
\partial_{t}\left(\alpha_{l} \rho_{l} U_{l}\right)+\nabla \cdot\left(\alpha_{l} \rho_{l} U_{l} \otimes U_{l}+\alpha_{l} P_{l} \mathcal{I}\right)-P_{I} \nabla\left(\alpha_{l}\right)=0 \\
\partial_{t}\left(\alpha_{l} E_{l}\right)+\nabla \cdot\left(\alpha_{l} U_{l}\left(E_{l}+P_{l}\right)\right)+P_{I} \partial_{t}\left(\alpha_{l}\right)=0 \\
\partial_{t}\left(\alpha_{g} \rho_{g}\right)+\nabla \cdot\left(\alpha_{g} \rho_{g} U_{g}\right)=0 \\
\partial_{t}\left(\alpha_{g} \rho_{g} U_{g}\right)+\nabla \cdot\left(\alpha_{g} \rho_{g} U_{g} \otimes U_{g}+\alpha_{g} P_{g} \mathcal{I}\right)-P_{I} \nabla\left(\alpha_{g}\right)=0 \\
\partial_{t}\left(\alpha_{g} E_{g}\right)+\nabla \cdot\left(\alpha_{g} U_{g}\left(E_{g}+P_{g}\right)\right)+P_{I} \partial_{t}\left(\alpha_{g}\right)=0
\end{array}\right.
$$

through the time interval $\left[t^{n}, t^{n}+\Delta t\right]$, with given initial values $W^{n}$, using a Finite Volume scheme to be defined. This provides a set of approximations $\tilde{W}$. 
- Relaxation step

Given discrete cell values of $\tilde{W}$, we compute approximations of the coupled set of ODEs corresponding to relaxation terms, that is:

$$
\left\{\begin{array}{l}
\partial_{t}\left(\alpha_{l}\right)=S_{1, l} \\
\partial_{t}\left(\alpha_{l} \rho_{l}\right)=0 \\
\partial_{t}\left(\alpha_{l} \rho_{l} U_{l}\right)=S_{3, l} \\
\partial_{t}\left(\alpha_{l} E_{l}\right)+P_{I} \partial_{t}\left(\alpha_{l}\right)=S_{4, l} \\
\partial_{t}\left(\alpha_{g} \rho_{g}+\alpha_{l} \rho_{l}\right)=0 \\
\partial_{t}\left(\alpha_{g} \rho_{g} U_{g}+\alpha_{l} \rho_{l} U_{l}\right)=0 \\
\partial_{t}\left(\alpha_{g} E_{g}+\alpha_{l} E_{l}\right)=0
\end{array}\right.
$$

\subsection{Computing the evolution step}

Many solvers have been proposed in the literature for such a purpose. Among those we may at least cite recent schemes based on approximate Riemann solvers, such as those detailed in $[31,35,36]$, but also algorithms that are presented in the papers $[1,2,7]$, which are grounded on the use of relaxation techniques. Approximate solutions in the evolution step may also be obtained using either the non-conservative form of Rusanov scheme, or the non-conservative form of the approximate Godunov scheme VFRoe-ncv [6]; we refer to $[11,10]$ for such a description. Obviously, the ultimate scheme has not been proposed yet. As it has been emphasized before, one must be aware here that the approximation of shock solutions makes sense for system (7), though the system has no conservative form, since first-order non-conservative products are only active in linearly degenerate fields. A straightforward consequence is that we expect schemes to converge towards the correct solution when the mesh is refined (see [17] for instance which examines this specific point while considering various choices of the closure law for $V_{I}$ ).

We will only recall here the non-conservative form of Rusanov scheme, for our system which may be written in a formal way:

$$
\partial_{t}(W)+\nabla \cdot(\mathcal{F}(W))+\mathcal{H}(W) \nabla \alpha=0
$$

If $\Delta t$ and $\operatorname{vol}\left(\Omega_{i}\right)$ respectively denote the time step and the volume of cell $\Omega_{i}$, and if $V(i)$ stands for the set of nodes $j$ neighbouring cell $i$, the scheme computes cell values $W_{i}^{n+1}$ in terms of $W_{j}^{n}$ following:

$$
\operatorname{vol}\left(\Omega_{i}\right)\left(W_{i}^{n+1}-W_{i}^{n}\right)+\Delta t \Sigma_{j \in V(i)}\left(f_{i j}^{R u s a n o v}\left(W_{i}^{n}, W_{j}^{n}, n_{i j}\right) S_{i j}\right)+\Delta t \mathcal{T}_{i}=0
$$

where the normal flux through the interface between cells $i$ and $j$, with surface $S_{i j}$ and outward normal vector $n_{i j}$ pointing from cell $i$ towards cell $j$, is:

$$
f_{i j}^{\text {Rusanov }}\left(W_{i}^{n}, W_{j}^{n}, n_{i j}\right)=\left(\left(\mathcal{F}\left(W_{i}^{n}\right)+\mathcal{F}\left(W_{j}^{n}\right)\right) \cdot n_{i j}-r_{i j}\left(W_{j}^{n}-W_{i}^{n}\right)\right) / 2
$$

The scalar $r_{i j}$ is equal to $\max \left(R_{i}^{n}, R_{j}^{n}\right)$, where $R_{k}^{n}$ is the spectral radius of the whole convection matrix associated with (9) and estimated at $W_{k}^{n}$. The non 
conservative term $\mathcal{T}_{i}$ is:

$$
\mathcal{T}_{i}=\mathcal{H}\left(W_{i}^{n}\right) \Sigma_{j \in V(i)} \bar{\alpha}_{i j} n_{i j} S_{i j}
$$

while setting: $\bar{\alpha}_{i j}=\left(\alpha_{i}+\alpha_{j}\right) / 2$. An advantage of this scheme is that:

\section{Property 4:}

Scheme (10) preserves positive values of partial masses $m_{\phi}$ and void fractions $\alpha_{\phi}$, provided that the following CFL-like condition holds on the time step $\Delta t$ :

$$
\Delta t \Sigma_{j \in V(i)}\left(r_{i j} S_{i j}\right)<2 \operatorname{vol}\left(\Omega_{i}\right)
$$

The proof is classical but is briefly recalled. Setting $\psi=m_{\phi}$, the cell scheme (10) enables to rewrite :

$\left.\operatorname{vol}\left(\Omega_{i}\right) \psi_{i}^{n+1}=\psi_{i}^{n}\left(\operatorname{vol}\left(\Omega_{i}\right)-\Delta t \Sigma_{j \in V(i)}\left(r_{i j} S_{i j}\right) / 2\right)+\Delta t \Sigma_{j \in V(i)}\left(\operatorname{coef}_{\psi}\right)_{i j}^{n}\left(\psi_{j}^{n}\right) S_{i j}\right)$

where all coefficients $\left(\operatorname{coe} f_{\psi}\right)_{i j}^{n}$ are positive. Thus $\psi_{i}^{n+1}$ is a convex combination of $\psi_{i}^{n}$ and neighbouring values $\psi_{j}^{n}$ if (13) holds. A similar calculation for $\psi=\alpha_{\phi}$ enables to finish the proof.

A drawback is that the scheme is less accurate than many approximate Riemann solvers. Nonetheless, as recalled in the last section, it enables to obtain convergent approximations when the mesh size diminishes (see [17, 13, 30] also for similar convergence studies).

We detail afterwards the relaxation step, with special focus on the pressure relaxation step which is rather tricky. The relaxation step is in fact split into three substeps through which one accounts for drag effects (velocity relaxation step), heat exchange (temperature relaxation step) and pressure relaxation effects respectively.

\subsection{Computing the velocity relaxation step}

This step accounts for drag terms only; it computes approximate values $Z^{n \text {,* }}$ of solutions of the system:

$$
\left\{\begin{array}{l}
\partial_{t}\left(\alpha_{l}\right)=0 \\
\partial_{t}\left(m_{l}\right)=0 \\
\partial_{t}\left(m_{l} U_{l}\right)=\mathcal{D} \\
\partial_{t}\left(\alpha_{l} E_{l}\right)=\left(U_{l}+U_{g}\right) \mathcal{D} / 2 \\
\partial_{t}\left(m_{g}+m_{l}\right)=0 \\
\partial_{t}\left(m_{g} U_{g}+m_{l} U_{l}\right)=0 \\
\partial_{t}\left(\alpha_{g} E_{g}+\alpha_{l} E_{l}\right)=0
\end{array}\right.
$$


through the following update:

$$
\left\{\begin{array}{l}
\alpha_{l}^{n, *}=\tilde{\alpha}_{l} \\
m_{\phi}^{n, *}=\tilde{m}_{\phi} \quad \text { for } \phi=l, g \\
U_{l}^{n, *}=\tilde{U}_{l}+\left(\tilde{U}_{g}-\tilde{U}_{l}\right) \frac{m_{g}^{n, *}\left(1-e^{\left(-\Delta t / \tau_{3}^{n}\right)}\right)}{m_{l}^{n, *}+m_{g}^{n, *}} \\
U_{g}^{n, *}=\tilde{U}_{g}-\left(\tilde{U}_{g}-\tilde{U}_{l}\right) \frac{m_{l}^{n, *}\left(1-e^{\left(-\Delta t / \tau_{3}^{n}\right)}\right)}{m_{n}^{n, *}+m_{g}^{n, *}} \\
\left(m_{l} e_{l}\right)^{n, *}=\tilde{m}_{l} \tilde{e}_{l}+\frac{m_{l}^{n, *} m_{g}^{n, *}}{m_{l}^{n, *}+m_{g}^{n, *}} \frac{\left(\tilde{U}_{l}-\tilde{U}_{g}\right)^{2}}{2}\left(1-e^{\left(-2 \Delta t / \tau_{3}^{n}\right)}\right) \\
\left(m_{g} e_{g}\right)^{n, *}=\tilde{m}_{g} \tilde{e_{g}}
\end{array}\right.
$$

\section{Proposition 1:}

The void fraction and the partial masses remain unchanged through this velocity relaxation step (16), and the total mean momentum and the mean total energy are also preserved. It may be easily checked that internal energies remain positive through this step.

The proof is obvious considering formula (16) and is left to the reader.

\subsection{Computing the temperature relaxation step}

Once again, both the void fraction and the partial masses remain steady through this step which computes approximations of solutions of system:

$$
\left\{\begin{array}{l}
\partial_{t}\left(\alpha_{l}\right)=0 \\
\partial_{t}\left(m_{l}\right)=0 \\
\partial_{t}\left(m_{l} U_{l}\right)=0 \\
\partial_{t}\left(\alpha_{l} E_{l}\right)=\left(\tau_{4}\right)^{-1} \frac{m_{l} C_{V, l} m_{g} C_{V, g}}{m_{l} C_{V, l}+m_{g} C_{V, g}}\left(T_{g}-T_{l}\right) \\
\partial_{t}\left(m_{g}+m_{l}\right)=0 \\
\partial_{t}\left(m_{g} U_{g}+m_{l} U_{l}\right)=0 \\
\partial_{t}\left(\alpha_{g} E_{g}+\alpha_{l} E_{l}\right)=0
\end{array}\right.
$$

We detail here the temperature relaxation scheme that updates internal energies according to the rule:

$$
\left\{\begin{array}{l}
\alpha_{l}^{n, * *}=\alpha_{l}^{n, *} \\
m_{\phi}^{n, * *}=m_{\phi}^{n, *} \quad \text { for } \phi=l, g \\
m_{\phi}^{n, * *} U_{,}^{n, * *}=m_{,}^{n, *} U_{\phi}^{n, *} \quad \text { for } \phi=l, g \\
m_{l}^{n, *}\left(e_{l}^{n, * *}-e_{l}^{n, *}\right)=\frac{\Delta t}{\theta^{n, *}}\left(T_{g}\left(e_{g}^{n, * *}, \rho_{g}^{n, *}\right)-T_{l}\left(e_{l}^{n, * *}, \rho_{l}^{n, *}\right)\right) \\
m_{g}^{n, *}\left(e_{g}^{n, * *}-e_{g}^{n, *}\right)+m_{l}^{n, *}\left(e_{l}^{n, * *}-e_{l}^{n, *}\right)=0
\end{array}\right.
$$

where $\theta^{n, *}=\left(\tau_{4}\right)^{n, *}\left(\frac{m_{l} C_{V, l}+m_{g} C_{V, g}}{m_{l} C_{V, l} m_{g} C_{V, g}}\right)^{n, *}$.

Remark 1:

This implicit algorithm guarantees the exact conservation of the total energy of the mixture. 
This step requires solving a non-linear system of two unknowns $\left(e_{g}^{n, * *}, e_{l}^{n, * *}\right)$, and we may give the following result:

\section{Proposition 2:}

We assume that, for $\phi=l, g$, the equations of state comply with:

$$
\partial_{e_{\phi}}\left(T_{\phi}\right)_{\mid \rho_{\phi}}>0 \quad \text { and } \quad: \lim _{e_{\phi} \rightarrow 0^{+}} T_{\phi}\left(e_{\phi}, \rho_{\phi}\right)=0 .
$$

Then system (18) admits a unique solution $\left(e_{g}^{n, * *}, e_{l}^{n, * *}\right)$, without any constraint on the time step $\Delta t$. Moreover, $e_{g}^{n, * *}$ and $e_{l}^{n, * *}$ respectively lie within intervals $\left[0, \frac{m_{g}^{n, *} e_{g}^{n, *}+m_{l}^{n, *} e_{l}^{n, *}}{m_{g}^{n, *}}\right]$ and $\left[0, \frac{m_{g}^{n, *} e_{g}^{n, *}+m_{l}^{n, *} e_{l}^{n, *}}{m_{l}^{n, *}}\right]$.

Proof:

We set: $X=e_{l}^{n, * *}$. Eliminating $e_{g}^{n, * *}$ from the last equation in (18), we need to find $X$ solution of the following equation:

$q(X) \stackrel{\text { def }}{=} m_{l}^{n, *}\left(X-e_{l}^{n, *}\right)-\frac{\Delta t}{\theta^{n, *}}\left(T_{g}\left(e_{g}^{n, *}-\frac{m_{l}^{n, *}\left(X-e_{l}^{n, *}\right)}{m_{g}^{n, *}}, \rho_{g}^{n, *}\right)-T_{l}\left(X, \rho_{l}^{n, *}\right)\right)=0$

Since :

$$
q^{\prime}(X)=m_{l}^{n, *}\left(1+\frac{\Delta t}{\theta^{n, *}}\left(\frac{1}{m_{g}^{n, *}} \partial_{e_{g}}\left(T_{g}\right)_{\mid \rho_{g}}+\frac{1}{m_{l}^{n, *}} \partial_{e_{l}}\left(T_{l}\right)_{\mid \rho_{l}}\right)\right)>0
$$

we know that $q(X)$ is increasing. Taking into account that:

$$
\lim _{X \rightarrow 0^{+}} q(X)=-m_{l}^{n, *} e_{l}^{n, *}-\frac{\Delta t}{\theta^{n, *}} T_{g}\left(e_{g}^{n, *}+\frac{m_{l}^{n, *} e_{l}^{n, *}}{m_{g}^{n, *}}, \rho_{g}^{n, *}\right)<0
$$

and:

$\lim _{X \rightarrow\left(e_{l}^{n, *}+m_{g}^{n, *} e_{g}^{n, *} / m_{l}^{n, *}\right)^{-}} q(X)=m_{g}^{n, *} e_{g}^{n, *}+\frac{\Delta t}{\theta^{n, *}} T_{l}\left(e_{l}^{n, *}+\frac{m_{g}^{n, *} e_{g}^{n, *}}{m_{l}^{n, *}}, \rho_{l}^{n, *}\right)>0$

we may conclude the proof of proposition 1: the equation $q(X)=0$ admits a unique solution within the range $\left[0, e_{l}^{n, *}+m_{g}^{n, *} e_{g}^{n, *} / m_{l}^{n, *}\right]$, and $e_{g}^{n, * *}$ may be deduced from:

$$
e_{g}^{n, * *}=e_{g}^{n, *}-m_{l}^{n, *}\left(X-e_{l}^{n, *}\right) / m_{g}^{n, *} .
$$

The proof provides a practical way to compute solutions of system (18), that is actually used in the code. We emphasize that other -simpler- algorithms may be exhibited (see appendix B for instance); however, practical computations seem to show that the non-linear temperature relaxation scheme described here provides better results (see [24] and section IV).

We turn now to the most difficult part which corresponds to the pressure relaxation step. 


\subsection{Computing the pressure relaxation step}

The pressure relaxation step computes approximations of solutions of the ODEs:

$$
\left\{\begin{array}{l}
\partial_{t}\left(\alpha_{l}\right)=S_{1, l} \\
\partial_{t}\left(m_{\phi}\right)=0 \text { for } \phi=l, g \\
\partial_{t}\left(m_{\phi} U_{\phi}\right)=0 \text { for } \phi=l, g \\
\partial_{t}\left(m_{l} e_{l}\right)+P_{l} \partial_{t}\left(\alpha_{l}\right)=0 \\
\partial_{t}\left(\alpha_{g} E_{g}+\alpha_{l} E_{l}\right)=0
\end{array}\right.
$$

Two distinct schemes have been proposed in [22] in order to obtain approximate solutions of (19).

The first one is a semi-implicit scheme, that is such that the existence and uniqueness of the discrete solution is ensured, whatever the equations of state would be. This first algorithm is described in [22]. We focus here on the second one which is totally implicit with respect to the unknown $\left(P_{l}, P_{g}, \alpha_{l}\right)$. It must be emphasized that both schemes guarantee positive values of void fractions $\alpha_{\phi}$ and a perfect balance of total energies.

The implicit pressure-relaxation scheme calculates $\left(P_{l}, P_{g}, \alpha_{l}\right)^{n, * * *}$, solution of the following step:

$$
\left\{\begin{array}{l}
\alpha_{l}^{n, * * *}-\alpha_{l}^{n, * *}=\frac{\Delta t}{\tau_{2}^{n, * *}} \alpha_{l}^{n, * * *} \alpha_{g}^{n, * * *}\left(P_{l}^{n, * * *}-P_{g}^{n, * * *}\right) /\left(\left|P_{l}^{n, * *}\right|+\left|P_{g}^{n, * *}\right|\right) \\
m_{\phi}^{n, * * *}=m_{\phi}^{n, * *} \text { for } \phi=l, g \\
m_{\phi}^{n, * * *} U_{\phi}^{n, * * *}=m_{\phi}^{n, * *} U_{\phi}^{n, * *} \quad \text { for } \phi=l, g \\
m_{l}^{n, * *}\left(e_{l}^{n, * * *}-e_{l}^{n, * *}\right)+P_{l}^{n, * * *}\left(\alpha_{l}^{n, * * *}-\alpha_{l}^{n, * *}\right)=0 \\
m_{g}^{n, * *}\left(e_{g}^{n, * * *}-e_{g}^{n, * *}\right)+m_{l}^{n, * *}\left(e_{l}^{n, * * *}-e_{l}^{n, * *}\right)=0
\end{array}\right.
$$

using notations: $e_{\phi}^{n, * * *}=e_{\phi}\left(P_{\phi}^{n, * * *}, \rho_{\phi}^{n, * * *}\right)$, and $\rho_{\phi}^{n, * * *}=m_{\phi}^{n, * * *} / \alpha_{\phi}^{n, * * *}$. Conditions that guarantee the existence and uniqueness of the solution $\left(P_{l}, P_{g}, \alpha_{l}\right)^{n, * * *}$ are given below.

\section{Property 5:}

Assume that both equations of state (EOS) for gas and liquid phase are perfect gas EOS, which means that: $\rho_{\phi} e_{\phi}\left(P_{\phi}, \rho_{\phi}\right)=P_{\phi} /\left(\gamma_{\phi}-1\right)$ for $\phi=g, l$, with $\gamma_{\phi}>$ 1. Then the scheme (20) admits a unique relevant solution $P_{l}^{n, * * *}, P_{g}^{n, * * *}, \alpha_{l}^{n, * * *}$, such that $P_{l}^{n, * * *}>0, P_{g}^{n, * * *}>0$ and $\alpha_{l}^{n, * * *}$ lies in $[0,1]$.

Proof: the proof is the same as the one detailed in [10]. Actually, pressure solutions can be expressed in terms of the main scalar unknown $X=1-\alpha_{l}^{n, * * *}$

$$
\left\{\begin{array}{l}
P_{l}^{n, * * *}(X)=\left(\gamma_{l}-1\right)\left(m_{l} e_{l}\right)^{n, * *} /\left(1-X+\left(\gamma_{l}-1\right)\left(\alpha_{g}^{n, * *}-X\right)\right) \\
P_{g}^{n, * * *}(X)=\left(\gamma_{g}-1\right)\left(\left(m_{g} e_{g}\right)^{n, * *}+\left(\alpha_{g}^{n, * *}-X\right) P_{l}^{n, * * *}(X)\right) / X
\end{array}\right.
$$


where the solution $X \in\left[0, \alpha_{m}\right]$ is the unique solution of the equation :

$$
H(X) \stackrel{\text { def }}{=} X(1-X)\left(P_{g}^{n, * * *}(X)-P_{l}^{n, * * *}(X)\right)-\frac{\theta}{\Delta t}\left(X-\alpha_{g}^{n, * *}\right)=0
$$

with $\alpha_{m}=\left(1+\left(\gamma_{l}-1\right) \alpha_{g}^{n, * *}\right) / \gamma_{l}$.

For some applications, it may be useful to apply for a real EOS for the liquid phase. Thus we turn to the case where the liquid EOS complies with standard constraints : $\partial_{P_{l}}\left(e_{l}\right)_{\mid \rho_{l}}\left(P_{l}, \rho_{l}\right)>0$, and $0 \leq P_{l}$. We have the following result:

\section{Proposition 3:}

Assume that the EOS of the gas phase is a perfect gas EOS: $\rho_{g} e_{g}\left(P_{g}, \rho_{g}\right)=$ $P_{g} /\left(\gamma_{g}-1\right)$. Define $\alpha_{M}$ the smallest value $x \in[0,1]$ solution of the equation $A(x)=0$ where :

$$
A(x) \stackrel{\text { def }}{=}\left(m_{l}\right)^{n, * *} \partial_{P_{l}}\left(e_{l}\right)_{\mid \rho_{l}}\left(p_{l}(x), \rho_{l}(x)\right)-x+\left(\alpha_{g}\right)^{n, * *}
$$

where: $\rho_{l}(x)=\left(m_{l}\right)^{n, * *} /(1-x)$, and $p_{l}(x)$ is solution of:

$$
\left(m_{l}\right)^{n, * *} e_{l}\left(p_{l}(x), \rho_{l}(x)\right)+p_{l}(x)\left(\alpha_{g}^{n, * *}-x\right)=\left(m_{l} e_{l}\right)^{n, * *}
$$

Then the scheme (20) admits a relevant solution $\left(P_{l}^{n, * * *}, P_{g}^{n, * * *}, \alpha_{l}^{n, * * *}\right)$ such that $\left(1-\alpha_{l}^{n, * * *}\right) \in\left[0, \alpha_{M}\right]$.

Proof: the main lines of the proof are given below. We still look for $x$ solution of the equation:

$$
h(x) \stackrel{\text { def }}{=} x(1-x)\left(p_{g}(x)-p_{l}(x)\right)-\frac{\theta}{\Delta t}\left(x-\alpha_{g}^{n, * *}\right)=0
$$

where :

$$
\left\{\begin{array}{l}
\rho_{l}(x)=\left(m_{l}\right)^{n, * *} /(1-x) \\
\left(m_{l}\right)^{n, * *} e_{l}\left(p_{l}(x), \rho_{l}(x)\right)+p_{l}(x)\left(\alpha_{g}^{n, * *}-x\right)=\left(m_{l} e_{l}\right)^{n, * *} \\
p_{g}(x)=\left(\gamma_{g}-1\right)\left(\left(m_{g} e_{g}\right)^{n, * *}+\left(\alpha_{g}^{n, * *}-x\right) p_{l}(x)\right) / x
\end{array}\right.
$$

Using the implicit definition of $p_{l}(x)$ in the second equation of $(24)$, a straightforward derivation shows that:

$$
A(x) p_{l}^{\prime}(x)=\left(\left(\rho_{l} c_{l}\right)^{2} \partial_{P_{l}}\left(e_{l}\right)_{\mid \rho_{l}}\right)\left(p_{l}(x), \rho_{l}(x)\right)>0
$$

Starting with the definition of $A(x)$ above, we note that:

$$
A(0)=\left(m_{l}\right)^{n, * *} \partial_{P_{l}}\left(e_{l}\right)_{\mid \rho_{l}}\left(p_{l}(0), \rho_{l}(0)\right)+\left(\alpha_{g}\right)^{n, * *}>0
$$

and:

$$
\lim _{x \rightarrow 1^{-}} A(x)=-1+\left(\alpha_{g}\right)^{n, * *}<0
$$


Thus the equation $A(x)=0$ admits at least one solution in $[0,1]$. Using the definition of $\alpha_{M}$, we know that $A\left(\alpha_{M}\right)=0$; thus :

$$
\alpha_{M}-\left(\alpha_{g}\right)^{n, * *}=\left(m_{l}\right)^{n, * *} \partial_{P_{l}}\left(e_{l}\right)_{\mid \rho_{l}}\left(p_{l}\left(\alpha_{M}\right), \rho_{l}\left(\alpha_{M}\right)\right)>0
$$

Moreover, we have:

$$
\begin{array}{r}
h(x)=-\frac{\theta}{\Delta t}\left(x-\alpha_{g}^{n, * *}\right)+\left(\gamma_{g}-1\right)\left(m_{g} e_{g}\right)^{n, * *}(1-x)+ \\
\left.(1-x) p_{l}(x)\left(\left(\gamma_{g}-1\right)\left(\alpha_{g}^{n, * *}-x\right)-x\right)\right)
\end{array}
$$

Thus: $h(0)=\alpha_{g}^{n, * *} \frac{\theta}{\Delta t}+\left(\gamma_{g}-1\right)\left(m_{g} e_{g}\right)^{n, * *}+\alpha_{g}^{n, * *}\left(\gamma_{g}-1\right) p_{l}(0)>0$. The function $p_{l}(x)$ is increasing within the interval $\left[0, \alpha_{M}\right]$, with $\lim _{x \rightarrow \alpha_{M}^{-}} p_{l}(x)=+\infty$; hence, we get that :

$$
\lim _{x \rightarrow \alpha_{M}^{-}} h(x)=-\infty
$$

We may eventually conclude that the equation $h(x)=0$ admits a solution in the interval $\left[0, \alpha_{M}\right]$.

Remark 2: the implicit scheme (20) is exactly the same as the one introduced for dense granular gas-particle flows in [10]. In that case, the counterpart of property 5 was obtained considering stiffened gas EOS within each phase (gas phase and particle phase). It must be also emphasized that we retrieve that $\alpha_{M}=\alpha_{m}$ when a perfect gas EOS is applied for the liquid phase.

Remark 3: the algorithm that was proposed in [11] in order to compute approximations of the pressure relaxation substep (19) does not ensure the exact conservation of the total energy of the mixture, which explains why we do not consider it herein.

\section{Numerical results}

In the first subsection, we focus on the computation of the evolution step involving all convective terms. Next we turn to the verification of the relaxation step. In each case, we examine rates of convergence in terms of the mesh size, with special emphasis on unsteady computations. The last subsection is devoted to the computation of a two-dimensional unsteady case in a square domain containing a heated wall boundary.

In this section, the following unities are used : $m$ for distances, $\mathrm{kg} / \mathrm{m}^{3}$ for densities, $\mathrm{m} / \mathrm{s}$ for velocities, $\mathrm{Pa}$ (Pascal) for pressures, $K$ (Kelvin) for temperatures, $s$ for times and $J / s / m^{2}$ for heating fluxes.

\subsection{Verification of the evolution step}

We use for this test case perfect gas EOS within each phase, setting $\gamma_{g}=1.4$ and $\gamma_{l}=1.1$. We show below a very simple Riemann problem associated with 
(7), considering the initial data $W_{L}, W_{R}$ given in table 1 . The solution contains

\begin{tabular}{|c||c|c|c|}
\hline & Left state $\left(W_{L}\right)$ & Intermediate state $\left(W_{\text {int }}\right)$ & Right state $\left(W_{R}\right)$ \\
\hline$\alpha_{l}$ & 0.95 & 0.05 & 0.05 \\
\hline$\rho_{l}$ & 1.0 & 0.9659 & 0.9659 \\
\hline$U x_{l}$ & -10.0 & 83.3542 & 83.3542 \\
\hline$P_{l}$ & $10^{5}$ & 95258.2695 & 95258.2695 \\
\hline$\rho_{g}$ & 0.1 & 1.0 & 0.95 \\
\hline$U x_{g}$ & -15 & -15 & -34.0783 \\
\hline$P_{g}$ & $10^{5}$ & 99782.7935 & 92867.1544 \\
\hline
\end{tabular}

Table 1: : Initial condition for the first Riemann problem and intermediate states.

many ghost waves, and intermediate states only differ through waves associated with the $U_{g}$ contact wave and to the $U_{g}+c_{g}$ GNL wave. The latter wave behaves as a shock wave in this case, in agreement with the entropy inequality (5). The intermediate state $W_{\text {int }}$ lies between the contact wave and the gas GNL wave $U_{g}+c_{g}$. The CFL is set to $1 / 2$, and five meshes with 50 up to 500000 regular cells are used. We plot first on Figure 1 void fractions, densities, velocities and pressures, focusing on the mesh containing 500000 cells. Then we give in Figure 2 the $L^{1}$ norm of the error. An asymptotic rate $1 / 2$ is expected for this scheme, owing to the occurence of the contact wave $U_{g}$. More precisely, for this particular case, we expect the rate of convergence of variables $\alpha_{l}, \rho_{l}, P_{l}, U_{l}, P_{g}, \rho_{g}$ to be close to $1 / 2$, whereas we expect order 1 for the gas velocity $U_{g}$ (since it is preserved through the gas contact wave). Numerical results for fine meshes are in agreement with that. Nonetheless, due to the small variation of $P_{g}$ through $\lambda=U_{g}$ (see table 1 ), the rate of convergence seems to be a bit higher than $1 / 2$; a better agreement is observed for the second test case, which has the same structure, but involves a stronger jump of $P_{g}$ through the gas contact wave. These results are in agreement with those of $[13,30]$.

The second test case is another Riemann problem taken from [13], where initial conditions are given in table 2. This Riemann problem also contains a contact wave associated with $U_{g}$, and a right-going gas shock wave. As in the previous test case, the contact wave is smeared on coarse meshes, as expected, while the approximate solution around the gas shock wave is correct. We retrieve here the order $1 / 2$ for $\rho_{l}, P_{l}, U_{l}, P_{g}, \rho_{g}$ and order 1 for $U_{g}$. The exact solution and the numerical approximation for the finest mesh (100000 cells) are plotted on Figure 3, and we give in Figure 4 the $L^{1}$ norm of the error.

The third test case of this section is fully described in [35] (see. table $1 \mathrm{p}$. 499). Initial condition have been gathered in table 3. It involves two perfect gas EOS such that: $\gamma_{g}=\gamma_{l}=1.4$. Similar test cases can also be found in [3]. Numerical results can be found on Figure 14 using meshes with 50, 500 and 


\begin{tabular}{|c||c|c|c|}
\hline & Left state $\left(W_{L}\right)$ & Intermediate state $\left(W_{\text {int }}\right)$ & Right state $\left(W_{R}\right)$ \\
\hline$\alpha_{l}$ & 0.95 & 0.05 & 0.05 \\
\hline$\rho_{l}$ & 1.0 & 0.9561 & 0.9561 \\
\hline$U x_{l}$ & 10.0 & -84.3587 & -84.3587 \\
\hline$P_{l}$ & $10^{5}$ & 95185.1407 & 95185.1407 \\
\hline$\rho_{g}$ & 0.1 & 0.15 & 0.1 \\
\hline$U x_{g}$ & 15 & 15 & -357.2995 \\
\hline$P_{g}$ & $10^{4}$ & 95044.7777 & 53462.6875 \\
\hline
\end{tabular}

Table 2: : Initial condition for the second Riemann problem and intermediate states.

5000 cells. The solution involves 6 waves, with a left-going gas rarefaction wave, a right-going gas shock wave, a left-going liquid shock wave and a rightgoing liquid rarefaction wave. The gas and liquid contact waves have opposite signs. Intermediate states can be found in [35]. Here again, the two contact waves approximations are obviously rather poor on coarse meshes. Nonetheless, approximate values of intermediate states are relevant on the mesh with 500 cells.

\begin{tabular}{|c||c|c|}
\hline & Left state $\left(W_{L}\right)$ & Right state $\left(W_{R}\right)$ \\
\hline$\alpha_{l}$ & 0.2 & 0.7 \\
\hline$\rho_{l}$ & 0.2 & 1.0 \\
\hline$U x_{l}$ & 0 & 0 \\
\hline$P_{l}$ & 0.3 & 1.0 \\
\hline$\rho_{g}$ & 1.0 & 1.0 \\
\hline$U x_{g}$ & 0 & 0 \\
\hline$P_{g}$ & 1.0 & 1.0 \\
\hline
\end{tabular}

Table 3: : Initial condition for the first Riemann problem described in [35] (table 1, p. 499).

\subsection{Verification of the relaxation step}

\subsubsection{Velocity relaxation substep}

Two different series of verification test cases have been considered in [24] for the velocity relaxation step. The first one refers to a constant time scale $\tau_{3}$. In that case, the scheme (16) is perfect, since it computes the exact value at time $t^{n+1}$. The second series involves time scales $\tau_{3}$ which vary as $d /\left|U_{g}-U_{l}\right|$. In that case, analytic solutions allow computing the true error occuring in the velocity relaxation step. Several examples can be found in [24], which confirm that a first-order rate of convergence is achieved. 


\subsubsection{Temperature relaxation substep}

We present below some tests corresponding to constant time scales $\tau_{4}$, when computing approximate solutions of (17) with the scheme (18). We consider perfect gas EOS with the parameters:

$$
C_{V, l}=4154.3 \mathrm{~J} / \mathrm{kg} / \mathrm{K} \text { and } C_{V, g}=1500.9 \mathrm{~J} / \mathrm{kg} / \mathrm{K} \text {. }
$$

Figure 5 shows the convergence rate when $\tau_{4}=10^{-5}$, using the following initial condition of table 4.

\begin{tabular}{|c|c|c|c|c|c|c|c|c|c|c|}
\hline$\alpha_{l}$ & $\rho_{l}$ & $U x_{l}$ & $U y_{l}$ & $U z_{l}$ & $T_{l}$ & $\rho_{g}$ & $U x_{g}$ & $U y_{g}$ & $U z_{g}$ & $T_{g}$ \\
\hline 0.5 & 998.3227 & 1 & 1 & 1 & 3.7008 & 0.5578 & 1 & 1.1 & 1.2 & 360.2211 \\
\hline
\end{tabular}

Table 4: : Initial condition for the temperature relaxation test case

The linear scheme [24] recalled in appendix B is optimal in the case of constant time scales $\tau_{4}$ with constant parameters $C_{V, \phi}$, unlike the non-linear scheme (18); actually, round-off errors are found for the linear scheme in that case. We note that the rate of convergence for scheme (18) is close to 1 , as expected.

\subsubsection{Pressure relaxation substep}

Eventually, we provide an example of measured convergence rates in the pressure relaxation step. This one is crucial, and should be handled with great care. Otherwise, both the present model (where the relaxation time scale $\tau_{2}$ is non-zero) and standard two-fluid models (corresponding to $\tau_{2}=0$ ) may be confused, if inadequate "rough" schemes are used to provide approximate solutions of (19). The initial conditions of the test case are given in table 5. Figure 6 and Figure 7 show the behaviour of the scheme (20) and the comparison with another half-implicit scheme introduced in [22] and recalled in [24], focusing on void fractions and pressures within each phase at time $t=10^{-5}$. The $L^{1}$ norm of the error has been plotted as a function of the time step, focusing on an analytic test case described in [22] for $\gamma_{l}=2, \gamma_{g}=3$ and $\tau_{2}=10^{-6}$. In all cases examined in the latter reference, the implicit scheme provides the best approximations, whatever the time step and the pressure relaxation time scale $\tau_{2}$ are.

\begin{tabular}{|c|c|c|c|c|c|c|c|c|c|c|}
\hline$\alpha_{l}$ & $\rho_{l}$ & $U x_{l}$ & $U y_{l}$ & $U z_{l}$ & $P_{l}$ & $\rho_{g}$ & $U x_{g}$ & $U y_{g}$ & $U z_{g}$ & $P_{g}$ \\
\hline 0.15 & 1000 & 0 & 0 & 0 & $210^{6}$ & 10 & 1 & 1.1 & 1.2 & $110^{5}$ \\
\hline
\end{tabular}

Table 5: : Initial condition for the pressure relaxation test case 
Remark 4: In reference [10], mesh refinement effects were examined when $\tau_{2}=\epsilon<<1$ and when $\tau_{2}=0$ respectively, using coarse and very fine onedimensional meshes (with up to $10^{6}$ cells). This enables to retrieve the fact that the initial-value problem associated with $\tau_{2}=0$ is ill-posed: spurious oscillations arise when the mesh size is sufficiently small, and this is particularly spectacular for void fraction profiles, since the algorithm guarantees bounded variations owing to properties 4 and 5 (see [21] and [17] also for a similar study).

\subsection{Two-dimensional numerical results}

We consider now the two-dimensional unsteady computation of a heated wall in an almost square domain, where the wall contains a small cavity in the middle of the lower part (see Figure 8). The computational domain contains $311 \times 10^{3}$ regular cells, and the CFL number is set to $1 / 2$. Homogeneous Neumann-type boundary conditions have been used on the left, right and upper boundaries. The relaxation time scales are constant in this experiment, and are defined in table 6 .

\begin{tabular}{|c|c|c|}
\hline$\tau_{2}$ & $\tau_{3}$ & $\tau_{4}$ \\
\hline $10^{-9}$ & $10^{-4}$ & $10^{-5}$ \\
\hline
\end{tabular}

Table 6: : Values of time scales in the 2D experiment.

Uniform initial conditions are such that the fluid is at rest at the beginning of the computation, which means that:

$$
\begin{gathered}
\rho_{l}(x, y, 0)=765,7 \text { and } \rho_{g}(x, y, 0)=76,6 \\
P_{l}(x, y, 0)=P_{g}(x, y, 0)=166,3 \times 10^{5} \\
U_{l}^{x}(x, y, 0)=U_{g}^{x}(x, y, 0)=U_{l}^{y}(x, y, 0)=U_{g}^{y}(x, y, 0)=0 .
\end{gathered}
$$

These initial conditions have been defined by enforcing a velocity, temperature and pressure equilibrium; they roughly correspond to those of the flow in the core of a pressurized water reactor in a nuclear power plant if the fluid is at rest. The liquid phase statistical fraction has been set to 0.95 , and we use perfect gas EOS within each phase, setting: $\gamma_{g}=1.4$ and $\gamma_{l}=1.038$. The normal heat flux is uniform along the wall direction, and its steady value is: $H F=10^{6}$. The final time of the computation is $T_{5}=1.5 \times 10^{-2}$, and the average time step at the end of the computation is approximately equal to $\Delta t=3 . \times 10^{-6}$.

Figure 9 shows the liquid pressure isolines at time $T_{1}=3.46 \times 10^{-3}$. An almost spherical wave propagates, starting from the small squared lower cavity, and interacts with the plane waves issuing from the horizontal wall boundary. The two peaks that can be observed in the cavity corners are not associated with numerical issues. They are due to the fact that the two corresponding cells 
receive heat fluxes from two faces, whereas all other cells have at most one face that is heated. On the contrary the two cells at the exit corners of the cavity do not receive any heat flux. Actually these cells do not have a face on the heated wall, they only touch the wall through one of their vertices. Consequently, when added to the bluff body effect, it can explain why the pressure does not increase in these cells as in the other "wall cells". At the end of the computation, the acoustic fast waves have moved out of the computational domain, as may be observed on Figure 12.

Density profiles for both liquid and gas phases have been plotted along the vertical y-direction (see Figure 10 and Figure 11), starting from the small cavity $(-0.25<y<0)$ and up to the top of the computational domain, at three distinct times $T_{1}=3.461 \times 10^{-3}$ (dotted line), $T_{2}=6.92 \times 10^{-3}$ (dashed line) and $T_{5}=1.5 \times 10^{-2}$ (plain line). The decrease of the density due to the sudden heating flux in the small cavity can be retrieved.

The computational results that are shown on the Figure 13 enable to observe the relative difference between gas and liquid velocities (bottom left). It can be noticed that the norm of the relative velocity is important near the wall, at the exit of the cavity and at the fast wave fronts. At these locations, convection effects are significant and overcome velocity relaxation effect.The increase of the pressures near the wall eject the fluids out of the cavity.

Pressure profiles along the same axis $x=2$ can be found on Figure 12. Actually, discrepancies between liquid and gas pressures are very low compared with the amplitude of pressure waves, we found that the relative difference $\left|P_{g}-P_{l}\right| /\left(P_{g}+P_{l}\right)$ was less than $10^{-12}$. This was actually more or less expected since the time step, which is governed by the CFL condition, is equal on the selected mesh to $2.810^{-6}$. It thus remains large compared to $\tau_{2}$. It also seems worth mentionning that almost similar results have been obtained while changing the pressure relaxation time step within the range $\tau_{2} \in\left[10^{-9}, 10^{-6}\right]$, keeping other relaxation time steps unchanged and using the same mesh.

Obviously, results are still sensitive to mesh refinement, and finer meshes may be used in a 2D framework in order to increase the accuracy. Nonetheless, one must be aware that the 3D counterpart of the present 2D mesh would contain more than 150 millions of cells, which is of course far beyond what one can afford in an industrial situation.

\section{Conclusion}

We have detailed in this paper a fractional step method that is used in order to compute approximations of a two-fluid model for gas-liquid flows, and we have given some measured rates of convergence within each step. Actually, the most 
difficult steps correspond to:

- (i) the computation of stable and accurate enough approximations of the homogeneous convective part,

- (ii) the approximation of solutions of the coupled set of ODEs which govern temperature and pressure relaxation effects.

The first item is well-known and many workers have been working hard in that direction; some interesting convective solvers are thus available, nonetheless all are penalized by rather weak convergence rates, owing to the occurence of the two distinct LD waves associated with $U_{g}$ and $U_{l}$, which is in addition combined with the velocity relaxation effects due to drag; thus important mesh refinements are mandatory in order to obtain almost converged approximations, and this also motivates the design of even more accurate Riemann solvers.

On the contrary, up to the authors, little attention has been paid to the stable and accurate numerical approximation of relaxation effects. This is an annoying point since the pressure relaxation step is a key feature in the approximation of the whole set of PDEs. Authors feel convinced that an increased effort in that direction is also necessary.

Numerical experiments that are similar to the two-dimensional case described in the last section have been performed, while accouting for mass transfer at the interface. In that case of course, the relaxation procedure includes another substep in order to discretize $\Gamma$ contributions (see [23]).

\section{Acknowledgments:}

Part of this work has been achieved in the framework of the NEPTUNE project, with financial support by CEA (Commissariat à l'Energie Atomique et aux Energies Alternatives), EDF, IRSN (Institut de Radioprotection et de Sureté Nucléaire) and AREVA-NP. 


\section{Appendix A: Entropy-consistent interfacial clo- sure laws}

A general form of the governing equations of the mean quantities in the twofluid model can be written as follows in the viscous case, in a one-dimensional framework:

$$
\begin{aligned}
& \partial_{t}\left(\alpha_{\phi}\right)+V_{I}(W) \partial_{x}\left(\alpha_{\phi}\right)=\phi_{\phi}(W) \\
& \partial_{t}\left(\alpha_{\phi} \rho_{\phi}\right)+\partial_{x}\left(\alpha_{\phi} \rho_{\phi} U_{\phi}\right)=\Gamma_{\phi}(W) \\
& \partial_{t}\left(\alpha_{\phi} \rho_{\phi} U_{\phi}\right)+\partial_{x}\left(\alpha_{\phi} \rho_{\phi} U_{\phi}^{2}\right)+\partial_{x}\left(\alpha_{\phi} P_{\phi}\right)-P_{I}(W) \partial_{x}\left(\alpha_{\phi}\right)+\partial_{x}\left(\alpha_{\phi} F_{\phi}^{v i s c}\right)=D_{\phi}(W)+\Gamma_{\phi}(W) \bar{U}_{i n t} \\
& \partial_{t}\left(\alpha_{\phi} E_{\phi}\right)+\partial_{x}\left(\alpha_{\phi} U_{\phi}\left(E_{\phi}+P_{\phi}\right)\right)+P_{I}(W) \partial_{t}\left(\alpha_{\phi}\right)+\partial_{x}\left(\alpha_{\phi} F_{\phi}^{v i s c} U_{\phi}\right)=\psi_{\phi}(W)+\bar{U}_{i n t} D_{\phi}(W)+\Gamma_{\phi}(W) \bar{H}_{i n t}
\end{aligned}
$$

where $F_{\phi}^{\text {visc }}=\kappa_{\phi} \partial_{x}\left(U_{\phi}\right)$ is the viscous flux, and $\Gamma_{\phi}(W), D_{\phi}(W)$ and $\psi_{\phi}(W)$ take interfacial mass transfer, drag effects and interfacial heat transfer into account. The contribution $\phi_{\phi}(W)$ arising in the governing equation of the statistical void fraction $\alpha_{\phi}$ is due to the statistical averaging ([20]) of the topological equation $([9])$. Obviously, we must enforce the following:

$$
\sum_{\phi=l, g} \Gamma_{\phi}(W)=0 \quad ; \quad \sum_{\phi=l, g} \psi_{\phi}(W)=0 \quad ; \quad \sum_{\phi=l, g} D_{\phi}(W)=0 \quad ; \quad \sum_{\phi=l, g} \phi_{\phi}(W)=0 .
$$

since these represent interfacial transfer terms. We have noted: $\bar{U}_{i n t}=\left(U_{l}+\right.$ $\left.U_{g}\right) / 2$ and $\bar{H}_{\text {int }}=U_{l} U_{g} / 2$; these enable to account for mass and momentum transfer terms in the governing equations of mean velocities and mean total energies. We now postulate that some convex combination for $V_{I}(W)$ in terms of $U_{l}, U_{g}$ has been prescribed, that is:

$$
V_{I}(W)=\xi(W) U_{l}+(1-\xi(W)) U_{g} .
$$

where $\xi(W)$ lies in $[0,1]$. We recall for instance that physically relevant functions $\xi(W)$ have been proposed in $[8,11]$. We wish now to determine admissible forms of interfacial transfer terms $\Gamma_{l}(W), \phi_{l}(W), \psi_{l}(W), D_{l}(W)$ and $P_{I}(W)$.

We recall that temperatures are given by: $1 / T_{\phi}=\partial_{P_{\phi}}\left(s_{\phi}\right) / \partial_{P_{\phi}}\left(e_{\phi}\right)$, and also that chemical potentials read: $\mu_{\phi}=e_{\phi}+P_{\phi} / \rho_{\phi}-T_{\phi} s_{\phi}$. We rewrite the interfacial pressure $P_{I}(W)$ as a convex combination of both pressures $P_{l}, P_{g}$, that is:

$$
P_{I}(W)=\mu(W) P_{l}+(1-\mu(W)) P_{g}
$$

and get the following result:

\section{Proposition:}

We define:

$$
\eta(W)=\sum_{\phi=l, g} m_{\phi} s_{\phi} \quad \text { and: } \quad f_{\eta}(W)=\sum_{\phi=l, g} m_{\phi} U_{\phi} s_{\phi}
$$


In the non-viscous limit case $\kappa_{\phi}=0$, smooth solutions $W$ of (25) comply with the following entropy equation:

$$
\begin{array}{r}
\partial_{t}(\eta(W))+\partial_{x}\left(f_{\eta}(W)\right)=\Gamma_{l}(W)\left(\mu_{g}(W) / T_{g}-\mu_{l}(W) / T_{l}\right) \\
+D_{l}(W)\left(U_{g}-U_{l}\right)\left(1 /\left(2 T_{g}\right)+1 /\left(2 T_{l}\right)\right) \\
+\psi_{l}(W)\left(T_{g}-T_{l}\right) /\left(T_{g} T_{l}\right)+ \\
+\phi_{l}(W)\left(P_{l}-P_{g}\right)\left(1 /\left(2 T_{g}\right)+1 /\left(2 T_{l}\right)\right)
\end{array}
$$

when:

$$
\left.\mu(W)=((1-\xi(W))) / T_{l} /((1-\xi(W))) / T_{l}+\xi(W) / T_{g}\right)
$$

Since all quantities: $T_{g}-T_{l}, U_{g}-U_{l}, P_{g}-P_{l}, \mu_{g}-\mu_{l}$ are independent, the following entropy-consistent closure laws simply arise:

$$
\begin{aligned}
& \Gamma_{l}(W)=K_{\Gamma}(W)\left(\mu_{g}(W) / T_{g}-\mu_{l}(W) / T_{l}\right) \\
& D_{l}(W)=K_{U}(W)\left(U_{g}-U_{l}\right) \\
& \psi_{l}(W)=K_{T}(W)\left(T_{g}-T_{l}\right) \\
& \phi_{l}(W)=K_{P}(W)\left(P_{l}-P_{g}\right)
\end{aligned}
$$

where $K_{\Gamma}(W), K_{U}(W), K_{T}(W), K_{P}(W)$ denote positive coefficient functions. These may be rewritten as follows:

$$
\begin{gathered}
K_{U}(W)=m_{l} m_{g} /\left(m_{l}+m_{g}\right) / \tau_{U}(W), \\
K_{T}(W)=m_{l} m_{g} C_{l-v} /\left(m_{l}+m_{g}\right) / \tau_{T}(W),
\end{gathered}
$$

and hence agree with the classical two-fluid literature [25]. A relevant choice for $K_{P}$ that preserves positive values of void fractions is:

$$
K_{P}(W)=\alpha_{l} \alpha_{g} /\left(\left|P_{l}\right|+\left|P_{g}\right|\right) / \tau_{P}(W) .
$$

Thus $K_{U}(W), K_{T}(W), K_{P}(W)$ have been replaced by their associated relaxation time scales: $\tau_{U}, \tau_{T}$ and $\tau_{P}$.

The closure law $((29))$ is exactly the one that has been introduced in $[8,11]$. All closure laws presented above may be used for a broader class of two-fluid models $([20])$. 


\section{Appendix B: Half-implicit temperature relax- ation scheme}

Starting from the system of equations (17), the half implicit temperature scheme is obtained by solving the linearized system:

$$
\left\{\begin{array}{l}
\partial_{t}\left(\alpha_{l}\right)=0 \\
\partial_{t}\left(\alpha_{l} \rho_{l}\right)=0 \\
\partial_{t}\left(\alpha_{l} \rho_{l} U_{l}\right)=0 \\
\partial_{t}\left(\alpha_{l} E_{l}\right)=\mathcal{C}_{m} \\
\partial_{t}\left(\alpha_{g} \rho_{g}\right)=0 \\
\partial_{t}\left(\alpha_{g} \rho_{g} U_{g}\right)=0 \\
\partial_{t}\left(\alpha_{g} E_{g}\right)=-\mathcal{C}_{m}
\end{array}\right.
$$

with

$$
\mathcal{C}_{m}=\left(\tau_{4}(0)\right)^{-1} \frac{m_{l} C_{V, l}(0) m_{g} C_{V, g}(0)}{m_{l} C_{V, l}(0)+m_{g} C_{V, g}(0)}\left(T_{g}-T_{l}\right) .
$$

This system corresponds to the system (17) in which the parameters $\tau_{4}$ and $C_{V, \phi}$ have been frozen a initial time. The solutions for the volume fraction, the densities and the velocities are obvious (they are the same than those for the system (17)).

We now turn to the energy equations. The first step consists in a prediction of the relative temperature $T_{R}=T_{g}-T_{l}$. By combining the energy equations of (30) and by replacing $C_{V, \phi}(t)=\partial e_{\phi} / \partial T_{\phi}$ by $C_{V, \phi}(0)$, which has been frozen in (30)), it can be shown that the equation on the relative temperature $T_{R}$ is:

$$
\partial_{t}\left(T_{R}\right)=-\frac{T_{R}}{\tau_{4}(0)}
$$

This equation can be exactly integrated in $T_{R}(t)=T_{R}(0) e^{-t / \tau_{4}(0)}$. This prediction is then used to integrate exactly the linearized liquid energy equation:

$$
m_{l}\left(e_{l}(t)-e_{l}(0)\right)=\frac{m_{l} C_{V, l}(0) m_{g} C_{V, g}(0)}{m_{l} C_{V, l}(0)+m_{g} C_{V, g}(0)} T_{R}(0)\left(1-e^{-t / \tau_{4}(0)}\right),
$$

and the gas internal energy is obtained according to the conservation of the total energy of the mixture:

$$
m_{g}\left(e_{g}(t)-e_{g}(0)\right)=-m_{l}\left(e_{l}(t)-e_{l}(0)\right) .
$$

Obviously, if the time scale $\tau_{4}$ is constant and if the fluids are ruled by perfect gas EOS with constant parameters, the systems (30) and (17) coincide, and consequently the solutions above are exact for the system (17).

Starting from given values $Z^{n, *}$ at time $t^{n}$, the scheme compute approximate solutions at time $t^{n, * *}=t^{n}+\Delta t$. They are the exact solution of the system 
(30) at time $t=\Delta t$ with the initial condition $Z^{n, *}$. It thus reads:

$$
\left\{\begin{array}{l}
\alpha_{l}^{n, * *}=\alpha_{l}^{n, *} \\
m_{l}^{n, * *}=m_{l}^{n, *} \\
m_{g}^{n, * *}=m_{g}^{n, *} \\
U_{l}^{n, * *}=U_{l}^{n, *} \\
U_{g}^{n, * *}=U_{g}^{n, *} \\
m_{l}^{n, * *} e_{l}^{n, * *}=m_{l}^{n, *} e_{l}^{n, *}+\frac{m_{l} C_{V, l}^{n, *} m_{g} C_{V, g}^{n, *}}{m_{l} C_{V, *}^{n, *}+m_{g} C_{V, g}^{n, *}}\left(T_{g}^{n, *}-T_{l}^{n, *}\right)\left(1-e^{-\Delta t / \tau_{4}^{n, *}}\right), \\
m_{g}^{n, * *} e_{g}^{n, * *}=m_{g}^{n, *} e_{g}^{n, *}+m_{l}^{n, *} e_{l}^{n, *}-m_{l}^{n, * *} e_{l}^{n, * *}
\end{array}\right.
$$

This scheme obviously preserves the total energy of the mixture. 


\section{References}

[1] Ambroso A., Chalons C., Coquel F., Galié T., "Relaxation and numerical approximation of a two-fluid two-pressure diphasic model ", Math. Model. and Numer. Anal., vol. 43(6), pp. 1063-1098, 2009.

[2] Ambroso A., Chalons C., Raviart P.A., "A Godunov-type method for the seven-equation model of compressible two-phase flow ", Computers and Fluids, available online, 2011.

[3] Andrianov N., Warnecke G., " The Riemann problem for the BaerNunziato two-phase flow model", J. Comp. Physics., vol. 195, pp. 434-464, 2004 .

[4] Baer M.R., Nunziato J.W., "A two-phase mixture theory for the deflagration to detonation transition (DDT) in reactive granular materials", Int. J. Multiphase Flow, vol. 12(6), pp. 861-889, 1986.

[5] Bdzil J.B., Menikoff R., Son S.F., Kapila A.K., Stewart D.S., "Two-phase modeling of a DDT in granular materials: a critical examination of modeling issues", Phys. of Fluids, vol. 11, pp. 378-402, 1999.

[6] Buffard T., Gallouët T., HÉrard J.-M., "A sequel to a rough Godunov scheme. Application to real gases ", Computers and Fluids, vol. 29(7), pp. 813-847, 2000.

[7] Chalons C., Coquel F., Kokh S., Spillane N. "Large time-step numerical scheme for the seven-equation model of compressible two-phase flows" in the Proceedings of FVCA6, Finite Volumes for Complex Applications VI, Prague, June 6-10, 2011.

[8] Coquel F., Gallouët T., Hérard J.M., Seguin N., "Closure laws for a two-fluid two-pressure model", C. R. Acad. Sci. Paris, vol. I-332, pp. 927-932, 2002.

[9] Drew D.A., Passman S.L., Theory of multi-component fluids, Applied Mathematical Sciences, vol. 135, Springer, 1999.

[10] Gallouët T., Helluy P., Hérard J.-M., Nussbaum J., "Hyperbolic relaxation models for granular flows", Math. Model. and Numer. Anal., vol.44(2), pp.371-400, 2010.

[11] Gallouët T., Hérard J.-M., Seguin N., "Numerical modelling of two phase flows using the two-fluid two-pressure approach", Math. Mod. Meth. in Appl. Sci., vol. 14(5), pp. 663-700, 2004.

[12] Gavrilyuk S., Saurel R., "Mathematical and numerical modelling of two phase compressible flows with inertia", J. Comp. Physics., vol. 175, pp. 326-360, 2002. 
[13] Girault L., Hérard J.-M., " A two-fluid hyperbolic model in a porous medium", Math. Model. and Numer. Anal., vol. 44(6), pp. 1319-1348, 2010 .

[14] Glimm J, Saltz D., Sharp D.H., "Two-phase flow modelling of a fluid mixing layer", J. Fluid Mech., vol. 378, pp. 119-143, 1999.

[15] Godunov S.K., "Finite difference method for numerical computation of discontinuous solutions of the equations of fluid dynamics", Mat. Sb., vol. 47, pp. 271-300, 1959.

[16] Guelfi A., Bestion D., Boucker M., Boudier P., Fillion P., Grandotto M., Hérard J.M., Hervieu E., Peturaud P., "Neptune: a new software platform for advanced nuclear thermal hydraulics", Nuclear Science Engineering, vol. 156, pp. 281-324, 2007.

[17] Guillemaud V., "Modélisation et simulation numérique d'écoulements diphasiques par une approche bifluide à deux pressions", PhD thesis, Université Aix-Marseille I, Marseille, France, 2007.

[18] HÉrard J.-M., "A three-phase flow model", Mathematical Computer Modelling, vol. 45, pp. 432-455, 2007.

[19] Hérard J.-M., "Un modèle hyperbolique diphasique bi-fluide en milieu poreux", Comptes-rendus Mécanique, vol. 336, pp. 650-655, 2008.

[20] HÉrard J.-M., "Une classe de modèles diphasiques bi-fluides avec changement de régime", internal EDF report H-I81-2010-0486-FR, in French, 2010.

[21] Hérard J.-M., Hurisse O., "A simple method to compute standard two-fluid models", Int. J. Comput. Fluid Dynamics, vol. 19(7), pp. 475$482,2005$.

[22] Hérard J.-M., Hurisse O., "Schémas d'intégration du terme source de relaxation des pressions phasiques pour un modèle bifluide hyperbolique", EDF report H-I81-2009-1514-FR, in French, 2009.

[23] HÉrard J.-M., Hurisse O., "Numerical modelling of water-vapour flows using the two-fluid approach", in preparation, 2011.

[24] Hurisse O., "Note de vérification d'un prototype de code multidimensionnel basé sur un modèle bifluide instationnaire", EDF report, to appear, 2011.

[25] Ishin M. Thermofluid dynamic theory of two-phase flow, Collection de la Direction des Etudes et Recherches d'Electricité de France, Collection Eyrolles, 1975.

[26] Ishin M., Hibiki T., Thermofluid dynamics of two-phase flow, Springer, 2006. 
[27] Kapila A.K., R. Menikoff R., Bdzil J.B., Son S.F., Stewart D.S., "Two-phase modeling of a DDT in granular materials: reduced equations", Phys. of Fluids, vol. 13, pp. 3002-3024, 2001.

[28] Kapila A.K., Son S.F., Bdzil J.B., Menikoff R., Stewart D.S., "Two-phase modeling of a DDT: structure of the velocity relaxation zone", Phys. of Fluids, vol. 9(12), pp. 3885-3897, 1997.

[29] Labois M., "Modélisation des déséquilibres mécaniques dans les écoulements diphasiques : Approches par relaxation et par modèle réduit", PhD thesis, Université Aix-Marseille I, Marseille, France, 2007.

[30] LiU Y., PhD thesis, Université Aix-Marseille I, Marseille, France, in preparation.

[31] Lowe C.A., "Two-phase shock-tube problems and numerical methods of solution", J. Comp. Physics., vol. 204, pp. 598-632, 2005.

[32] Papin M., Abgrall R., Fermetures entropiques pour les modèles bifluides à sept équations, Comptes-Rendus Mécanique, vol. 333, pp. 838-842, 2005 .

[33] Ransom V., Hicks D.L., "Hyperbolic two-pressure models for two-phase flow", J. Comp. Physics., vol. 53, pp. 124-151, 1984.

[34] Saurel R., Abgrall R., "A Multiphase Godunov Method for Compressible Multifluid and Multiphase Flows ", J. Comp. Physics., vol. 150, pp. 425-467, 1999.

[35] Schwendeman D.W., Wahle C.W., Kapila A.K., "The Riemann problem and a high-resolution Godunov method for a model of compressible two-phase flow", J. Comp. Physics., vol. 212, pp. 490-526, 2006.

[36] Tokareva S.A., Toro E.F., "HLLC type Riemann solver for the BaerNunziato equations of compressible two-phase flow", J. Comp. Physics., vol. 229, pp. 3573-3604, 2010. 

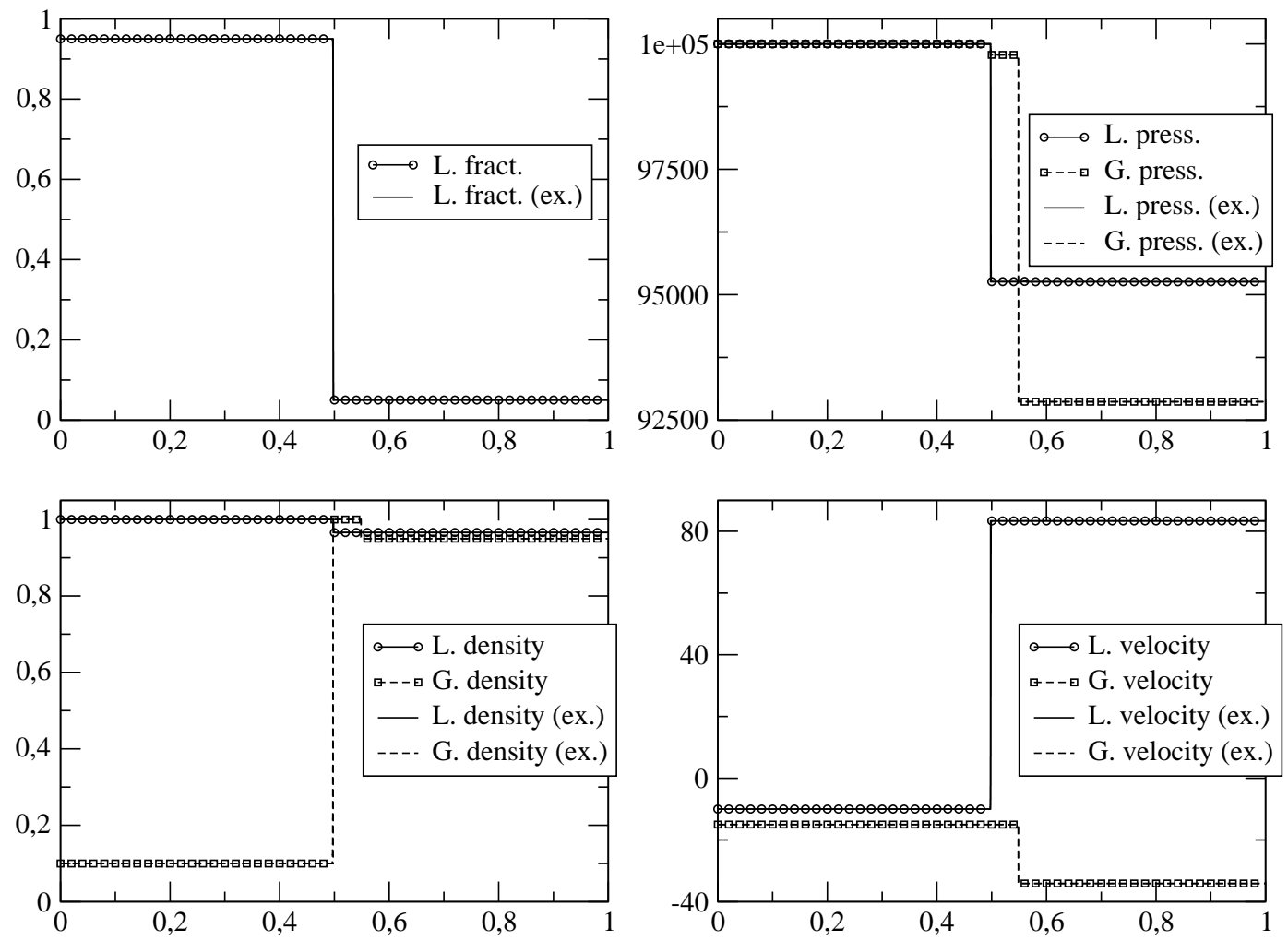

Figure 1: Approximate solution of the first Riemann problem obtained with 500000 cells and the exact solution (ex.) at time $t=1.410^{-4}$. Top left: liquid fraction, top right: pressures, bottom left: densities, bottom right: velocities. 


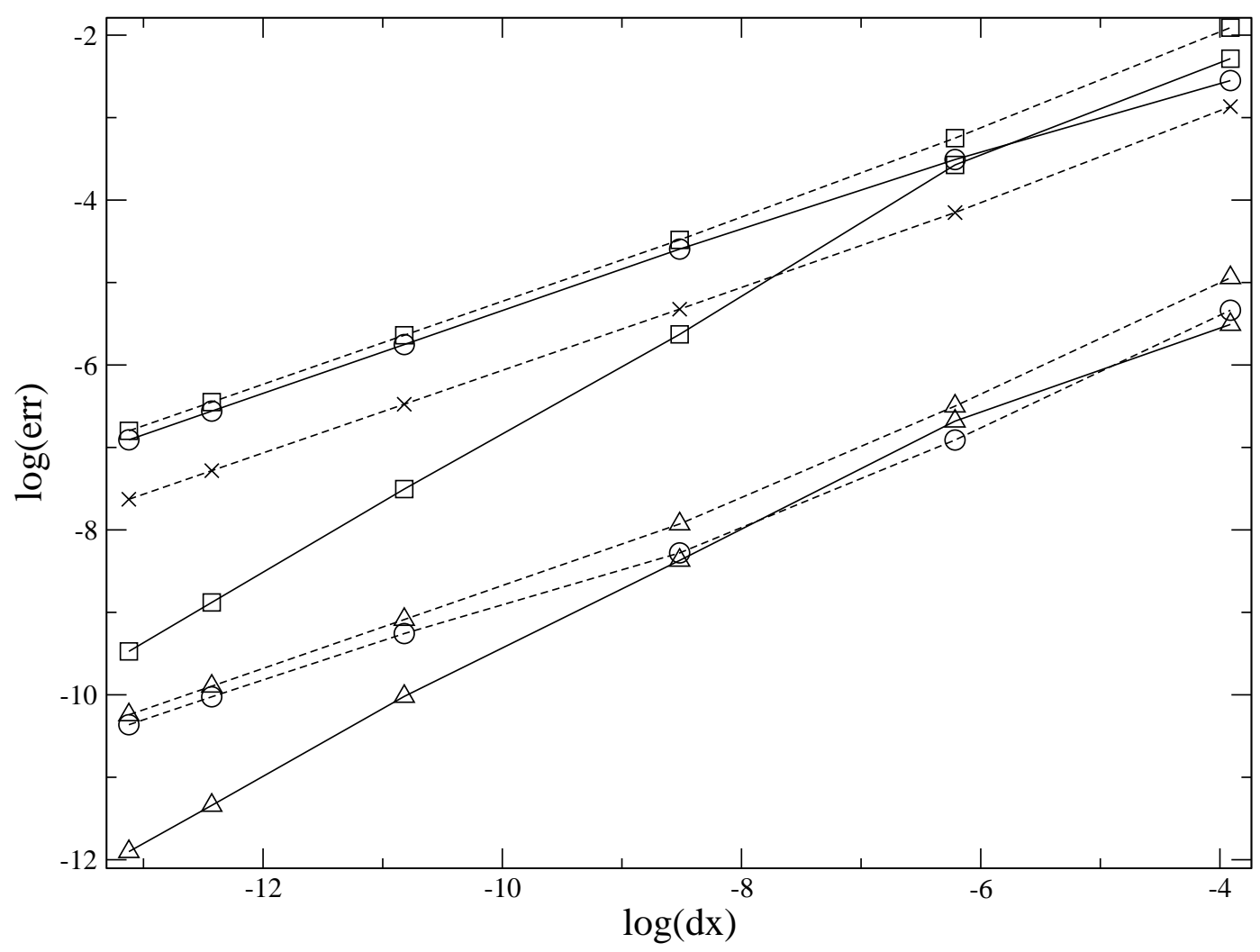

Figure 2: L1 norm of the error for the first Riemann problem. Plain lines: gas, dotted lines: liquid. Liquid mass fraction (crosses), velocities (squares), pressures (triangles), densities (circles). Meshes contain 500000, 250000, 50000, 5000,500 and 50 regular cells. 

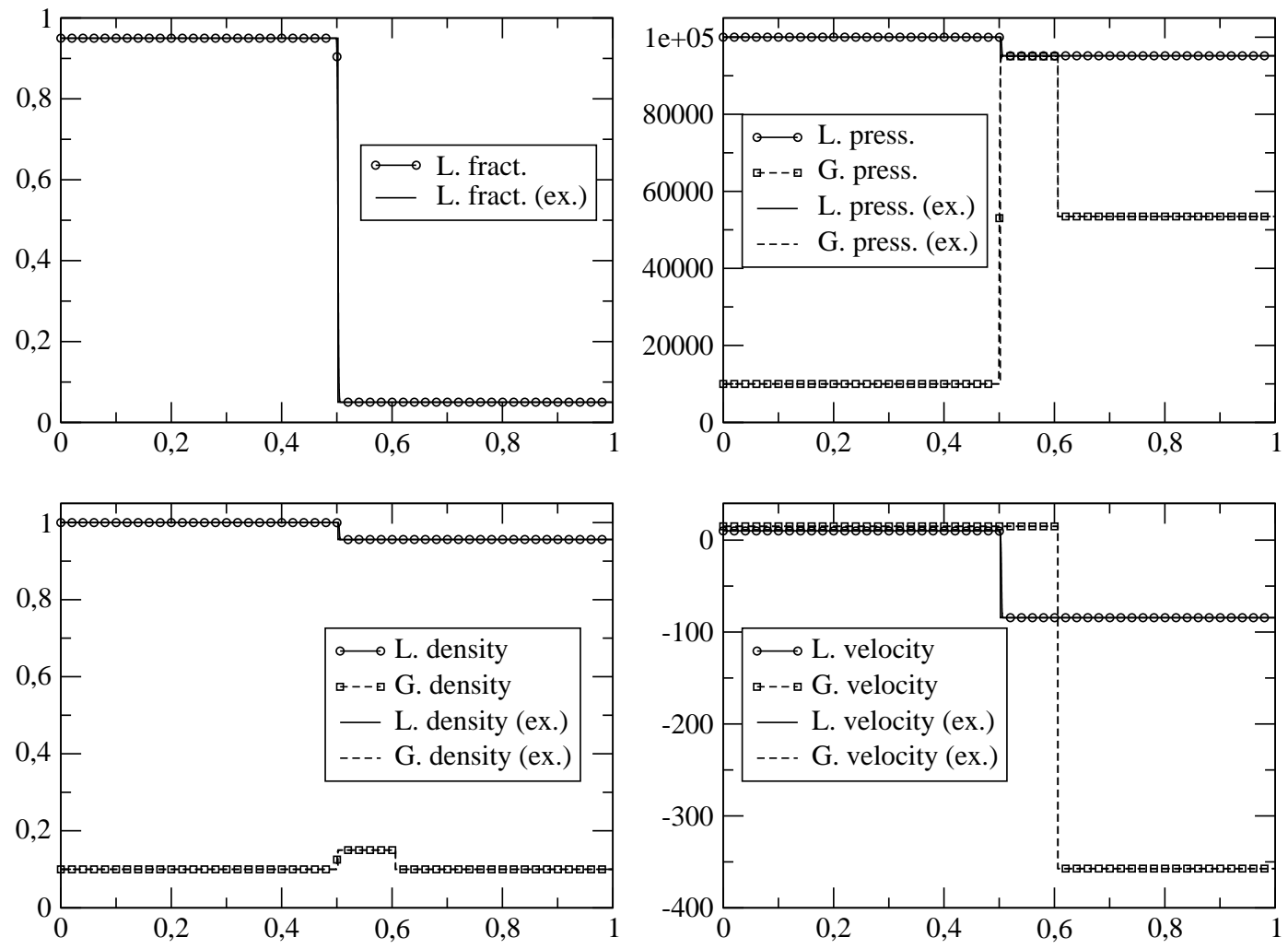

Figure 3: Approximate solution of the second Riemann problem obtained with 100000 cells and the exact solution (ex.) at time $t=1.410^{-4}$. Top left: liquid fraction, top right: pressures, bottom left: densities, bottom right: velocities. 


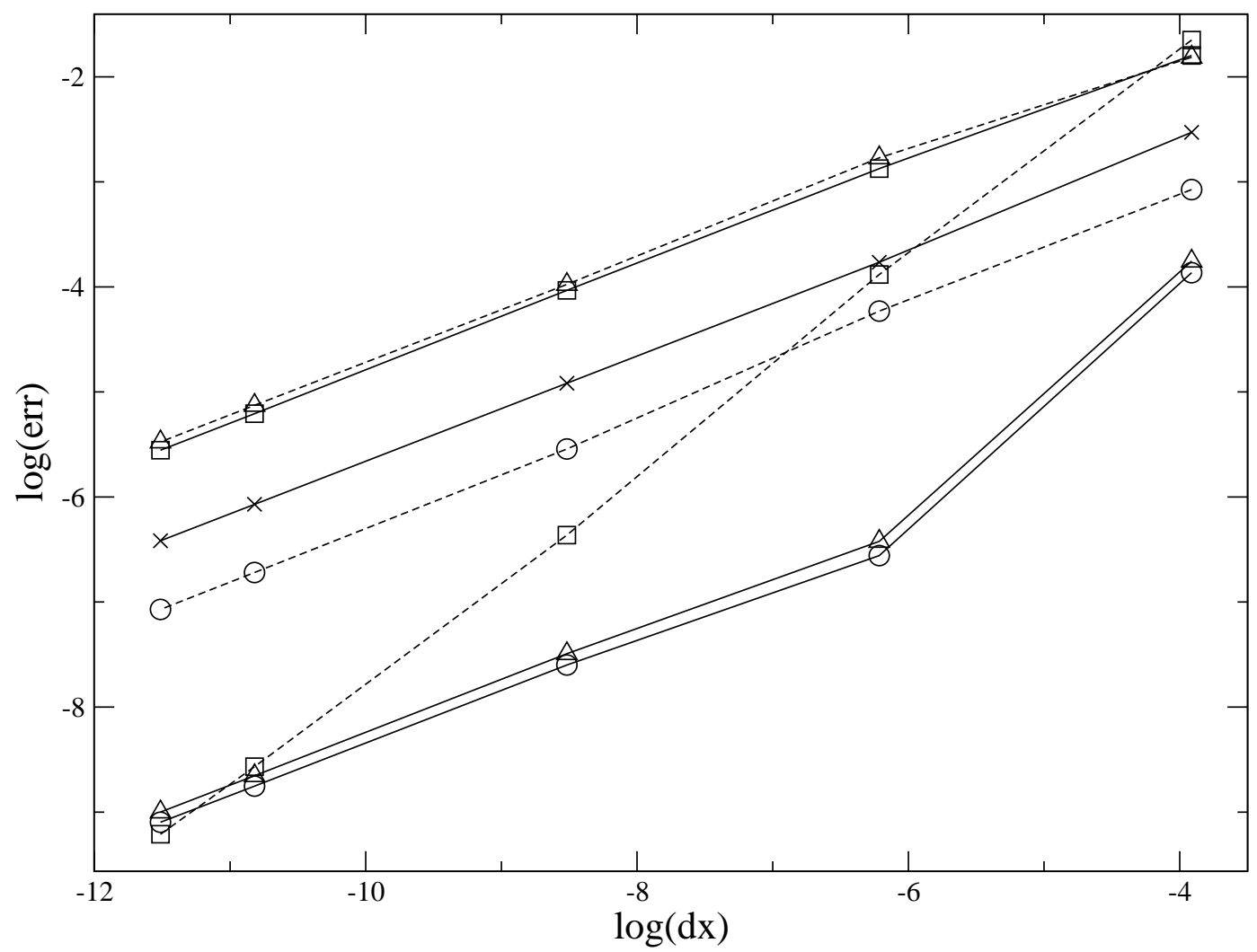

Figure 4: L1 norm of the error for the second Riemann problem. Plain lines: liquid, dotted lines: gas. Liquid mass fraction (crosses), velocities (squares), pressures (triangles), densities (circles). Meshes contain 100000, 50000, 5000, 500 and 50 regular cells. 


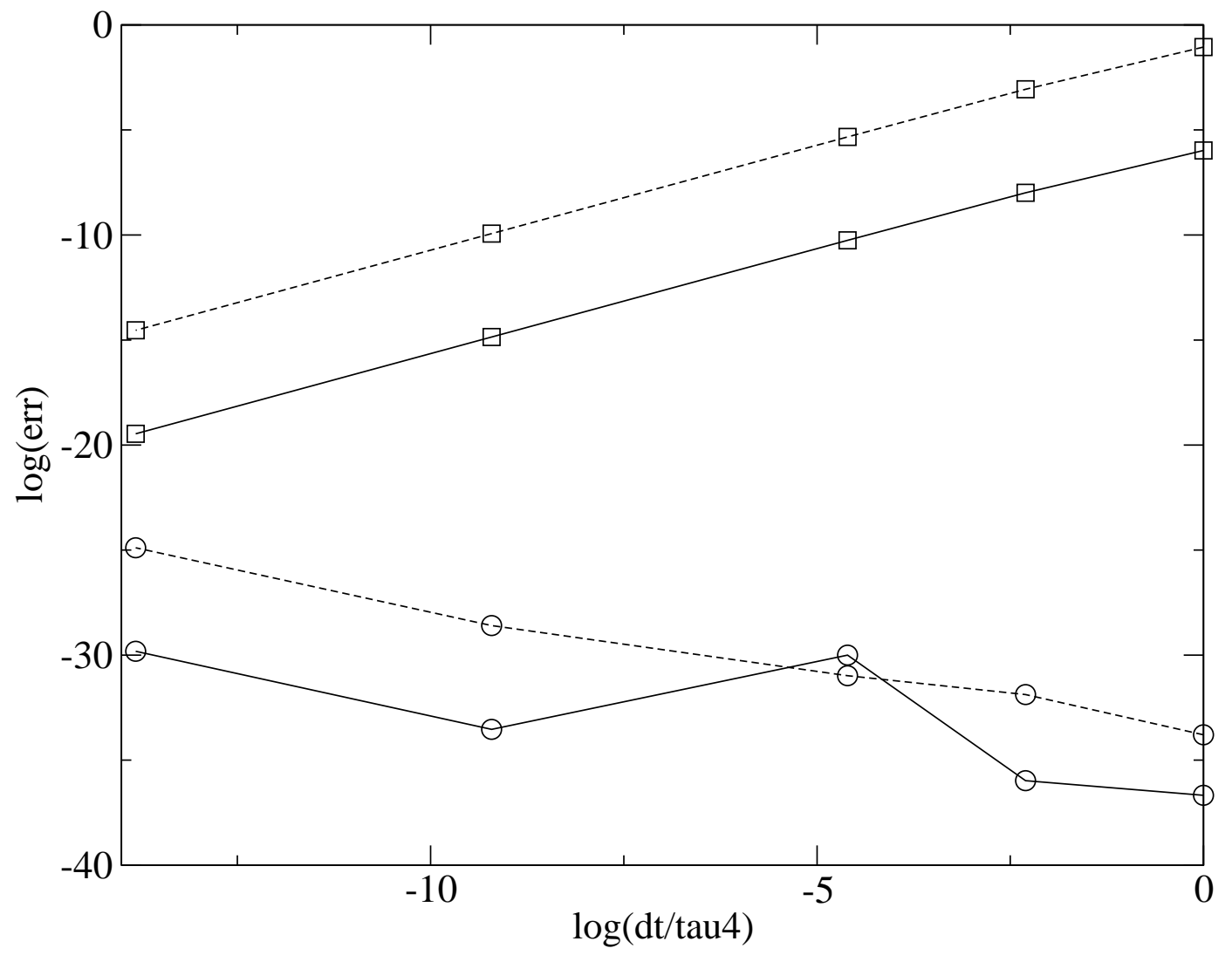

Figure 5: Temperature relaxation substep: error at time $T=10^{-5}$ as a function of $\Delta t / \tau_{4}=\left\{1,10^{-1}, 10^{-2}, 10^{-4}, 10^{-6}\right\}$. Liquid temperature (straight line), gas temperature (dotted line). Linear scheme (circles) versus non-linear scheme (squares). 


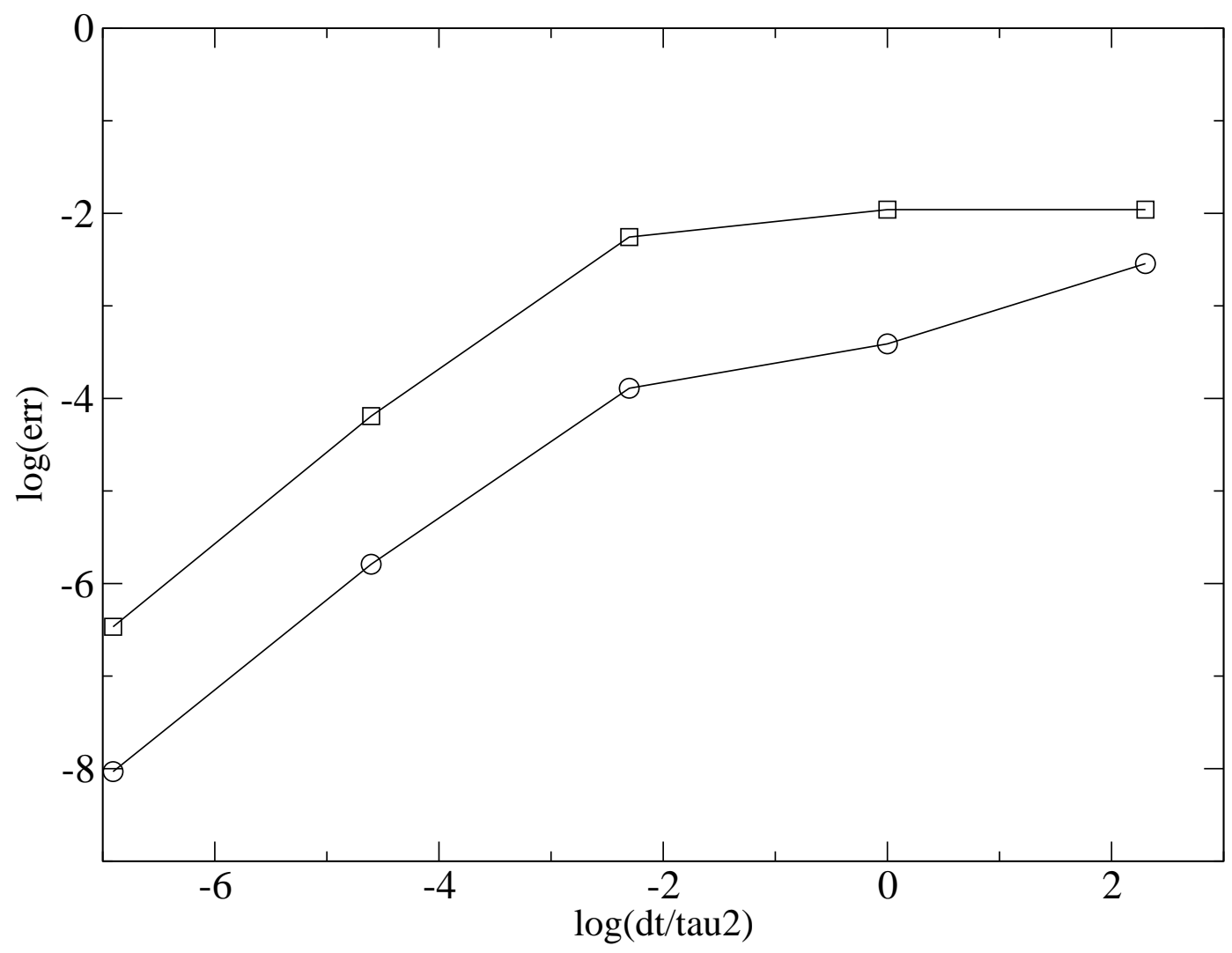

Figure 6: Pressure relaxation substep: measured L1 norm of the error for the void fraction at time $T=10^{-5}$ as a function of $\Delta t / \tau_{2}=$ $\left\{10,1,10^{-1}, 10^{-2}, 10^{-3}\right\}$. Implicit scheme (20) (circles) versus half-implicit scheme (squares). 


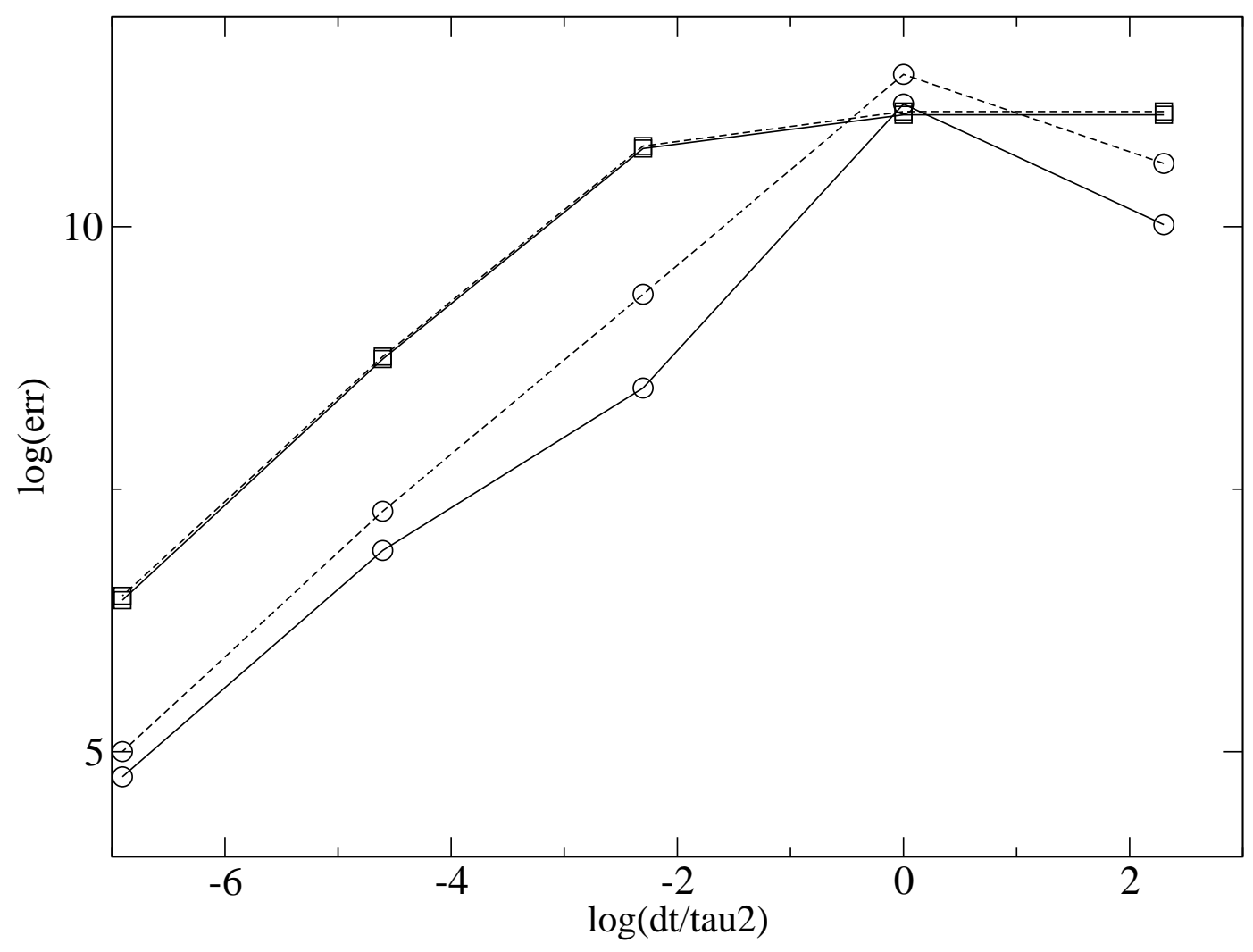

Figure 7: Pressure relaxation substep: measured L1 norm of the error for the liquid pressure (straight line) and the gas pressure (dotted line) at time $T=$ $10^{-5}$ as a function of $\Delta t / \tau_{2}=\left\{10,1,10^{-1}, 10^{-2}, 10^{-3}\right\}$. Implicit scheme (20) (circles) versus half-implicit scheme (squares). 


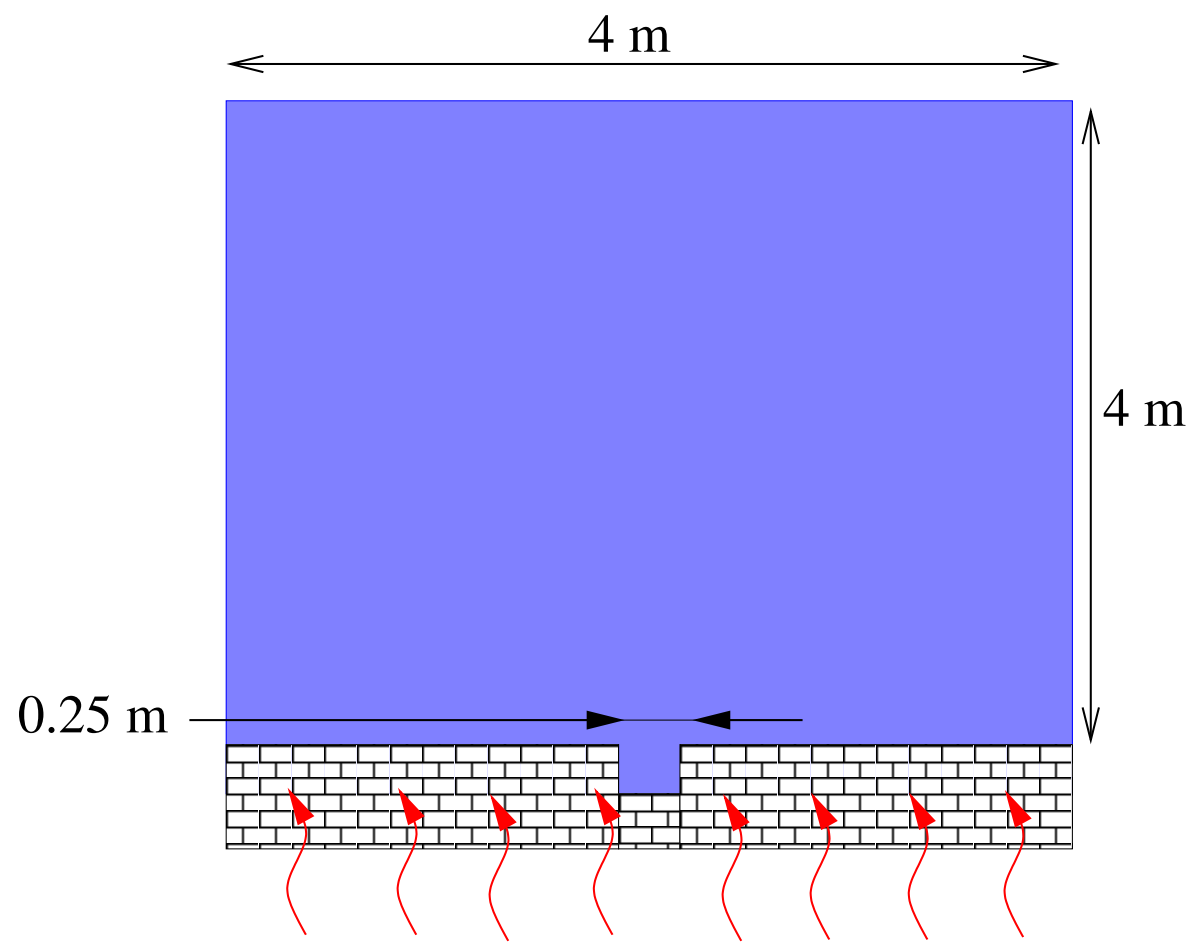

Figure 8: Heated wall: sketch of the computational domain 


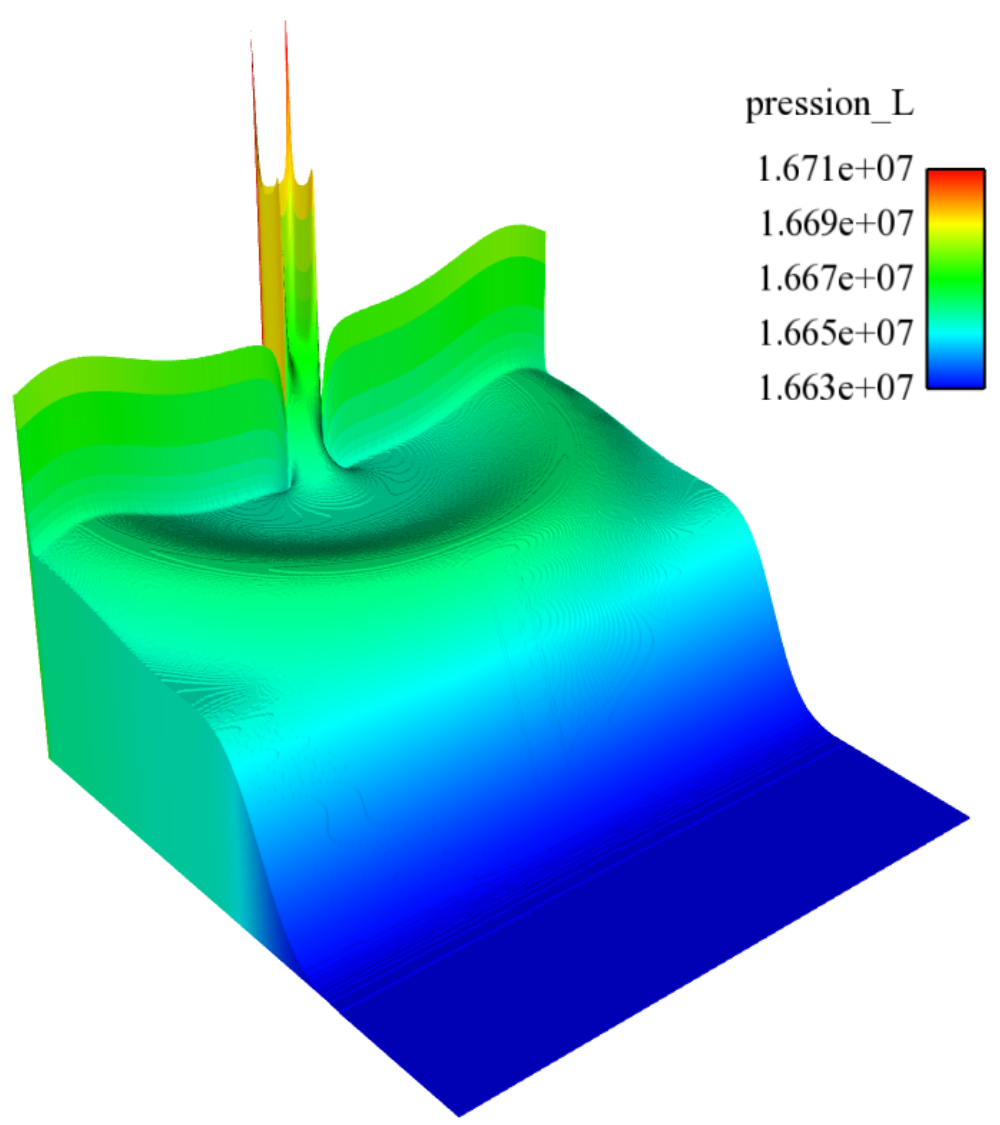

Figure 9: Heated wall: contours of the liquid pressure $P_{L}$ in the computational domain at time $T=T 1$. 


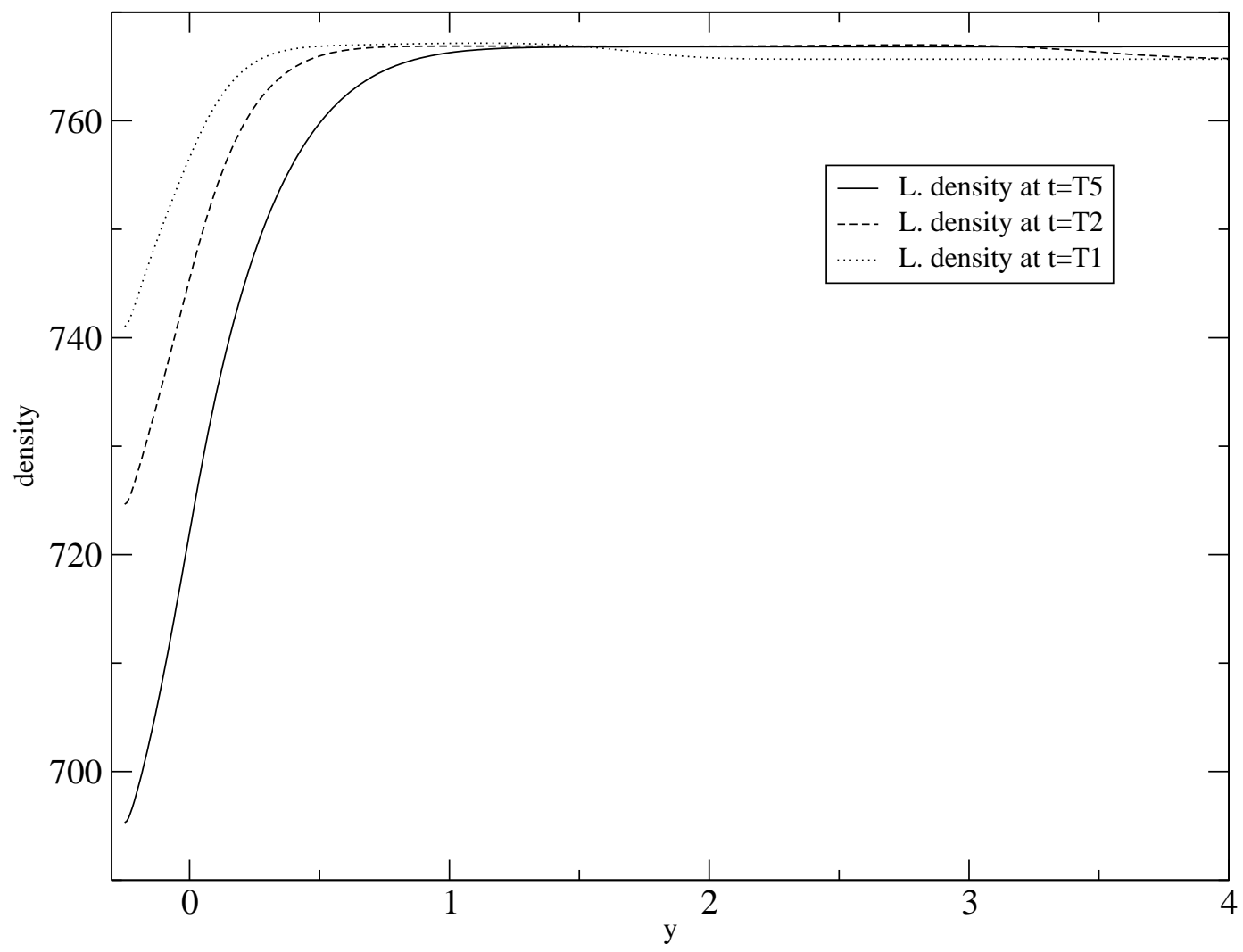

Figure 10: Heated wall: y-profile of the liquid density $\rho_{l}$ at three distinct times $T=T 1$ (dotted line), $T 2$ (dashed line), $T 5$ (plain line), at: $x=2$. The small cavity in the wall boundary corresponds to $-0.25<y<0$. 


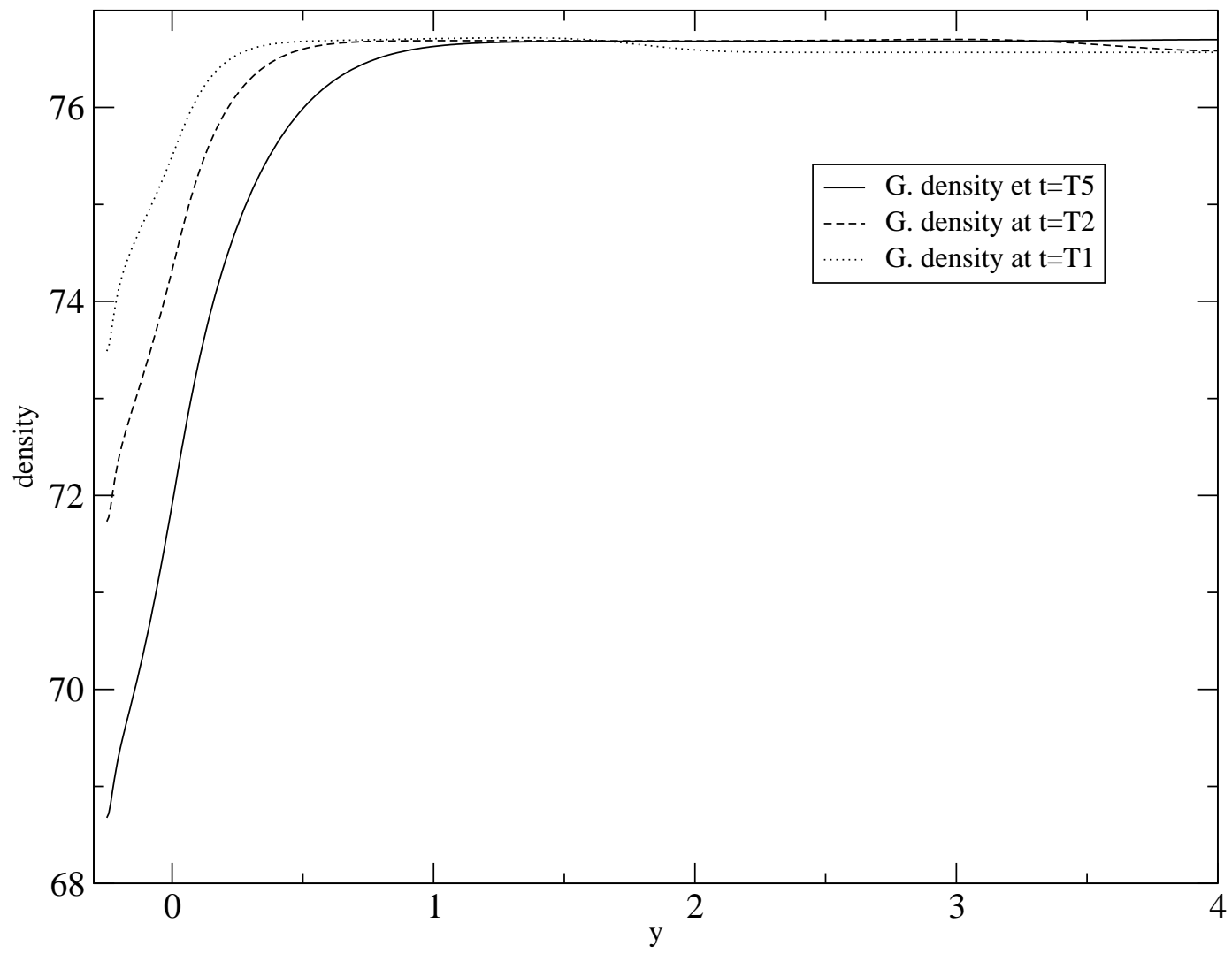

Figure 11: Heated wall: y-profile of the gas density $\rho_{g}$ at three distinct times $T=T 1$ (dotted line), $T 2$ (dashed line), $T 5$ (plain line), at: $x=2$. The small cavity in the wall boundary corresponds to $-0.25<y<0$. 


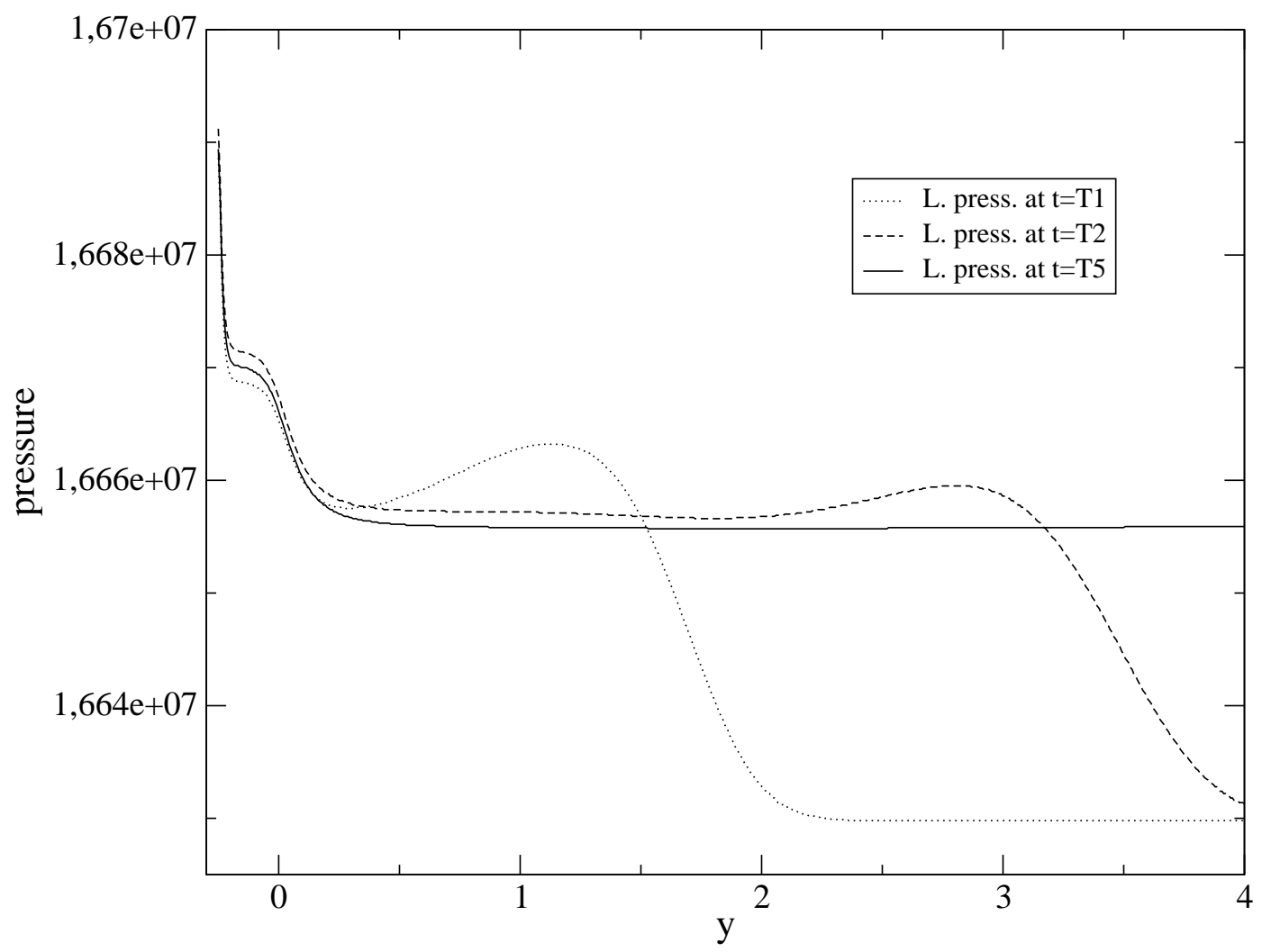

Figure 12: Heated wall: y-profiles of gas and liquid pressures $P_{g}, P_{l}$ at three distinct times $T=T 1$ (dotted line), $T 2$ (dashed line), $T 5$ (plain line), at: $x=2$. The small cavity in the wall boundary corresponds to $-0.25<y<0$. 

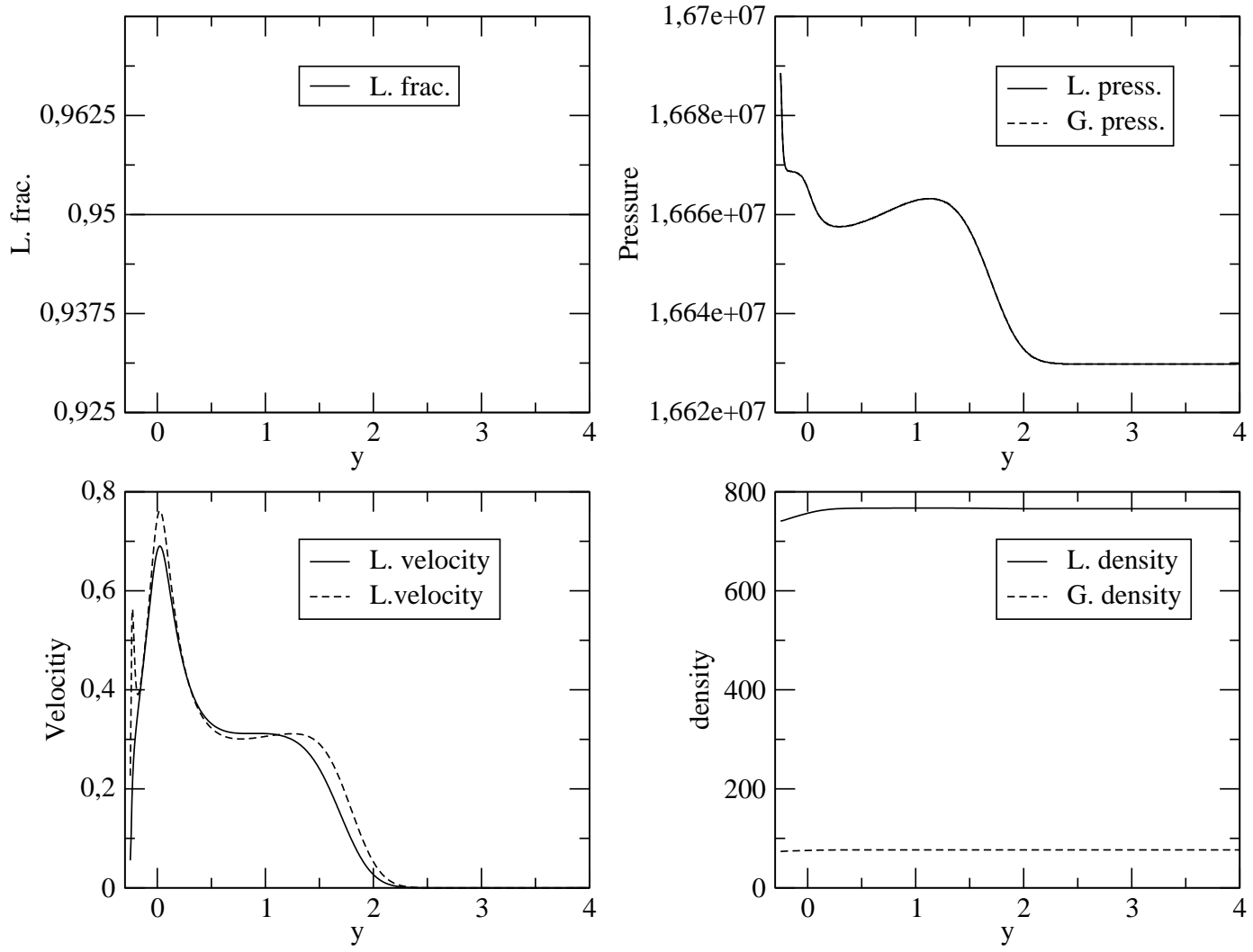

Figure 13: Heated wall: y-profiles at $x=2$ for the liquid void fraction (top left), pressures (top right), velocities in the y-direction (bottom left) and densities (bottom right) at time $T=T 1$. Liquid phase: plain line, gas phase: dashed line. 

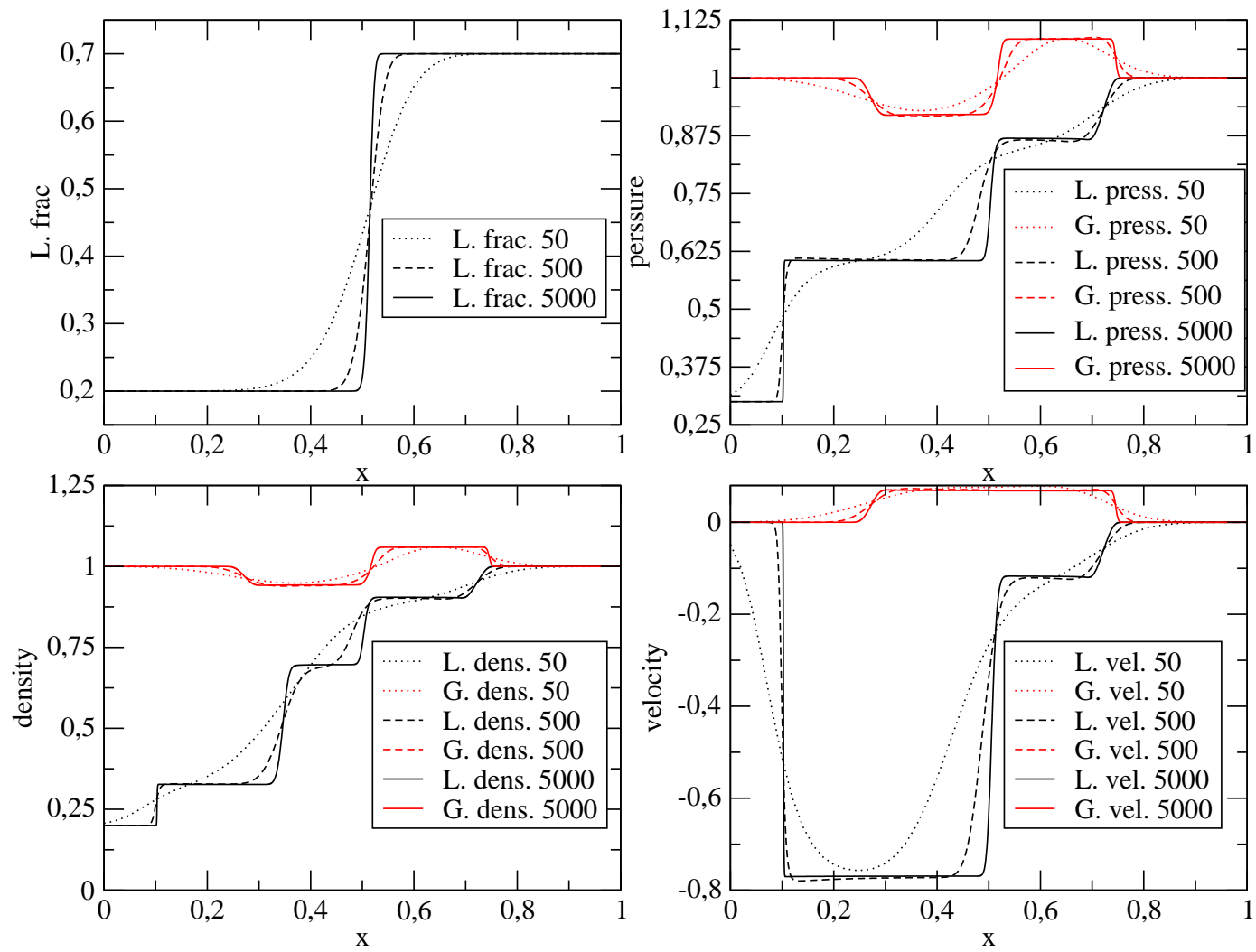

Figure 14: Approximate solution of the first Riemann problem described in [35] (table 1, p. 499) for 50, 500 and 5000 cells. 